\title{
Simulating propagation of decoupled elastic waves using low-rank approximate mixed-domain integral operators for anisotropic media
}

\author{
Jiubing Cheng ${ }^{1}$, Tariq Alkhalifah², Zedong $\mathrm{Wu}^{2}$, Peng Zou ${ }^{3}$, and Chenlong Wang ${ }^{3}$
}

\begin{abstract}
In elastic imaging, the extrapolated vector fields are decoupled into pure wave modes, such that the imaging condition produces interpretable images. Conventionally, mode decoupling in anisotropic media is costly because the operators involved are dependent on the velocity, and thus they are not stationary. We have developed an efficient pseudospectral approach to directly extrapolate the decoupled elastic waves using low-rank approximate mixed-domain integral operators on the basis of the elastic displacement wave equation. We have applied $k$-space adjustment to the pseudospectral solution to allow for a relatively large extrapolation time step. The low-rank approximation was, thus, applied to the spectral operators that simultaneously extrapolate and decompose the elastic wavefields. Synthetic examples on transversely isotropic and orthorhombic models showed that our approach has the potential to efficiently and accurately simulate the propagations of the decoupled quasi-P and quasi-S modes as well as the total wavefields for elastic wave modeling, imaging, and inversion.
\end{abstract}

\section{INTRODUCTION}

Multicomponent seismic data are increasingly acquired on land and at the ocean bottom in an attempt to better understand the geologic structure and characterize oil and gas reservoirs. Seismic modeling, reverse time migration (RTM), and full-waveform inversion (FWI) in areas with complex geology all require high-accuracy numerical algorithms for time extrapolation of waves. Because seismic waves propagate through the earth as a superposition of $\mathrm{P}$ - and $\mathrm{S}$-wave modes, an elastic wave equation is usually more accurate for wavefield extrapolation than an acoustic wave equation. Wave mode decoupling can not only help elastic imaging to produce physically interpretable images, which characterize reflectivities of various reflection types (Wapenaar et al., 1987; Dellinger and Etgen, 1990; Yan and Sava, 2008), but it can also provide more opportunity to mitigate the parameter trade-offs in elastic waveform inversion (Wang and Cheng, 2015).

For isotropic media, far-field $\mathrm{P}$ and $\mathrm{S}$ waves can be separated by taking the divergence and curl in the extrapolated elastic wavefields (Aki and Richards, 1980; Sun and McMechan, 2001). Alternatively, Ma and Zhu (2003) and Zhang et al. (2007) extrapolate vector P and $\mathrm{S}$ modes separately in an elastic wavefield by decomposing the wave equation into $\mathrm{P}$ - and $\mathrm{S}$-wave components. In the meantime, decoupling of the wave modes yields familiar scalar wave equations for the P and S modes (Aki and Richards, 1980). In anisotropic media, one cannot derive explicit single-mode time-space-domain differential wave equations so simply. Generally, P and S modes do not respectively polarize parallel and perpendicular to the wave vectors, and thus they are called quasi-P (qP) and quasi-S (qS) waves. They cannot be fully decoupled with divergence and curl operations (Dellinger and Etgen, 1990).

Anisotropic wave propagation can be formally decoupled in the wavenumber domain to yield single-mode pseudodifferential equations (Liu et al., 2009). Unfortunately, these equations in the timespace domain cannot be solved with traditional numerical schemes unless further approximation to the dispersion relation or phase velocity is applied (Etgen and Brandsberg-Dahl, 2009; Chu et al., 2011; Fowler and King, 2011; Zhan et al., 2012; Song and Alkhalifah, 2013; Du et al., 2014; Wu and Alkhalifah, 2014). To avoid solving the pseudodifferential equation, Xu and Zhou (2014) propose a nonlinear wave equation for pseudoacoustic qP-wave with an auxiliary scalar operator depending on the material parameters and the phase direction of the propagation at each spatial location. All these efforts are restricted to pure-mode scalar waves and do not honor the elastic effects such as mode conversion. Cheng and Kang (2014) and Kang and Cheng (2012) propose approaches to propa-

\footnotetext{
Manuscript received by the Editor 18 March 2015; revised manuscript received 21 October 2015; published online 15 March 2016.

${ }_{1}^{1}$ Tongji University, State Key Laboratory of Marine Geology, Shanghai, China. E-mail: cjb1206@tongji.edu.cn.

${ }^{2}$ King Abdullah University of Science and Technology, Thuwal, Saudi Arabia. E-mail: tariq.alkhalifah@kaust.edu.sa; zedong.wu@kaust.edu.sa.

Tongii University, Shanghai, China. E-mail: 1533006@tongji.edu.cn; skoltyz.10000@163.com.

(C) 2016 Society of Exploration Geophysicists. All rights reserved.
} 
gate the partially decoupled wave modes using the so-called pseudo pure-mode wave equations, and then they obtain completely decoupled $\mathrm{qP}$ or $\mathrm{qS}$ waves by correcting the polarization deviation of the pseudo pure-mode wavefields. Their approaches honor the kinematics of all wave modes but may distort the reflection/transmission coefficients if high contrasts exist in the velocity fields.

Alternatively, many have developed approaches to decouple the $\mathrm{qP}$ - and $\mathrm{qS}$-wave modes from the extrapolated elastic wavefields. Dellinger and Etgen (1990) generalize the divergence and curl operations to anisotropic media by constructing separators as polarization projection in the wavenumber domain. To tackle heterogeneity, these mode separators are rewritten by Yan and Sava (2009) as nonstationary spatial filters determined by the local polarization directions. Zhang and McMechan (2010) propose a wavefield decomposition method to separate elastic wavefields into vector $\mathrm{qP}$ and $\mathrm{qS}$ wavefields for vertically transverse isotropic (VTI) media. Accordingly, Cheng and Fomel (2014) propose fast mixeddomain algorithms for mode separation and vector decomposition in heterogeneous anisotropic media by applying low-rank approximation to the involved Fourier integral operators (FIOs) of the general form.

The motivation of this study is to develop an efficient approach to propagate and decouple the elastic waves for general anisotropic media. The primary strategy is to merge the numerical solutions for time extrapolation and vector decomposition into a unified Fourier integral framework and speed up the solutions using the lowrank approximation. This paper is organized as follows: We first demonstrate a pseudospectral solution to extrapolate the elastic displacement wavefields in the time domain. Then, we propose to merge the spectral operations for time extrapolation into the integral framework for vector decomposition of the wave modes. Applying low-rank approximations to the involved mixed-domain matrices, we obtain an efficient algorithm for simultaneous propagating and decoupling the elastic wavefields. We demonstrate the validity of the proposed method using 2D and 3D synthetic examples on the transversely isotropic (TI) and orthorhombic models with increasing complexity.

\section{PROPAGATING COUPLED ELASTIC WAVEFIELDS}

Following Carcione (2007), we denote the spatial variables $x, y$, and $z$ of a right-hand Cartesian system by the indices $i, j, \ldots=1,2$, and 3, respectively, the position vector by $\mathbf{x}$, a partial derivative with respect to a variable $x_{i}$ with $\partial_{i}$, and the first and second time derivatives with $\partial_{t}$ and $\partial_{t t}$, respectively. Matrix transposition is denoted by the superscript $T$. We also denote $\sqrt{-1}$ by $i$, the scalar and matrix products by the symbol ".", and the dyadic product by the symbol " $\otimes$ ". The Einstein convention of repeated indices is assumed unless otherwise specified.

\section{Pseudospectral solution of the elastic wave equation}

Wave propagation in general anisotropic elastic media is governed by the linearized momentum balance law and a linear constitutive relation between the stress and strain tensors. These governing equations can be combined to write the displacement formalism as follows:

$$
\rho \partial_{t t}^{2} \mathbf{u}=\nabla \cdot\left[\mathbf{C} \cdot\left(\nabla^{T} \cdot \mathbf{u}\right)\right]+\mathbf{f},
$$

where $\mathbf{u}=\left(u_{x}, u_{y}, u_{z}\right)^{T}$ represents the vector wavefields, $\mathbf{f}$ is the body force vector per unit volume, $\mathbf{C}$ is the $6 \times 6$ elasticity matrix representing the stiffness tensor with the Voigt's menu, and the spatial differential operator $\nabla$ has the following matrix representation:

$$
\nabla=\left(\begin{array}{cccccc}
\partial_{x} & 0 & 0 & 0 & \partial_{z} & \partial_{y} \\
0 & \partial_{y} & 0 & \partial_{z} & 0 & \partial_{x} \\
0 & 0 & \partial_{z} & \partial_{y} & \partial_{x} & 0
\end{array}\right)
$$

The pseudospectral method calculates the spatial derivatives using the fast Fourier transform (FFT), while approximating the temporal derivative with a finite difference (FD). Neglecting the source term, equation 1 is rewritten in the spatial Fourier domain for a homogeneous medium as follows:

$$
\partial_{t t}^{2} \hat{\mathbf{u}}+\boldsymbol{\Gamma} \hat{\mathbf{u}}=\mathbf{0}
$$

where $\hat{\mathbf{u}}$ is the wavefields in the wavenumber domain, $\mathbf{k}=$ $\left(k_{x}, k_{y}, k_{z}\right)$ is the wavenumber vector, and $\boldsymbol{\Gamma}=1 / \rho \mathbf{L} \cdot \mathbf{C} \cdot \mathbf{L}^{T}$ represents the $3 \times 3$ density normalized Christoffel matrix (as shown in Appendix A) with the wavenumber-domain counterpart of the space differential operator (removing the imaginary unit $i$ ) satisfies the following equation:

$$
\mathbf{L}=\left(\begin{array}{cccccc}
k_{x} & 0 & 0 & 0 & k_{z} & k_{y} \\
0 & k_{y} & 0 & k_{z} & 0 & k_{x} \\
0 & 0 & k_{z} & k_{y} & k_{x} & 0
\end{array}\right) .
$$

To calculate the second-order temporal derivatives, we use the standard leapfrog scheme, i.e.,

$$
\partial_{t t}^{2} u_{i}^{(n)}=\frac{u_{i}^{(n+1)}-2 u_{i}^{(n)}+u_{i}^{(n-1)}}{\Delta t^{2}},
$$

where $\Delta t=t^{n+1}-t^{n}$ is the time step. For constant-density homogeneous media, applying the two-step time-marching scheme leads to the following pseudospectral formula:

$$
\partial_{t t}^{2} \mathbf{u}^{(n)}=\boldsymbol{\Psi} \mathbf{u}^{(n)},
$$

with the spectral operator defined with the following kernel:

$$
\boldsymbol{\Psi}:=(2 \pi)^{-3} \iint \boldsymbol{\Gamma}(\mathbf{k}) e^{i \mathbf{k} \cdot(\mathbf{x}-\mathbf{y})} d \mathbf{y} d \mathbf{k} .
$$

Phase terms in the integral operator can be absorbed into forward and inverse Fourier transforms. This implies that the wavefields are first transformed into the wavenumber domain using forward FFTs, multiplied with the corresponding components of the Christoffel matrix, and finally transformed back into the space domain using inverse FFTs. For locally smooth media, we use a spatially varying Christoffel matrix to tackle the heterogeneity; i.e.,

$$
\boldsymbol{\Psi}:=(2 \pi)^{-3} \iint \boldsymbol{\Gamma}(\mathbf{x}, \mathbf{k}) e^{i \mathbf{k} \cdot(\mathbf{x}-\mathbf{y})} d \mathbf{y} d \mathbf{k} .
$$

The extended formation of this pseudospectral elastic wave propagator is shown in Appendix B. Spectral methods are characterized by the use of Fourier basis functions to describe the field variables and have an advantage over FD schemes in that the mesh 
requirements are more relaxed (Kosloff et al., 1989; Liu and Li, 2000).

\section{Adjustment to the pseudospectral solution}

In general, the two-step time-marching pseudospectral solution is limited to a small time step because larger time steps lead to numerical dispersion and stability issues. At higher computational costs, high-order FD (Dablain, 1986) can be applied to address this difficulty. As an alternative to second-order temporal differencing, a time integration technique based on rapid expansion method can provide higher accuracy with less computational efforts (Kosloff et al., 1989). As Du et al. (2014) demonstrate, one-step time-marching schemes (Zhang and Zhang, 2009; Sun and Fomel, 2013), especially using optimized polynomial expansion, usually give more accurate approximations to heterogeneous extrapolators for larger time steps. In this section, we discuss a strategy to extend the time step for the previous two-step time-marching pseudospectral scheme according to the eigenvalue decomposition of the Christoffel matrix.

Because the Christoffel matrix is symmetric positive definite, it has a unique eigen decomposition of the following form:

$$
\boldsymbol{\Gamma}=\sum_{i=1}^{3} \lambda_{i}^{2} \mathbf{a}_{i} \otimes \mathbf{a}_{i}
$$

where $\lambda_{i}^{2}$ are the eigenvalues and $\mathbf{a}_{i}$ are the eigenvectors of $\boldsymbol{\Gamma}$ with $\mathbf{a}_{i} \cdot \mathbf{a}_{j}=\delta_{i j}$. The three eigenvalues correspond to phase velocities of the three-wave modes with $\lambda_{i}=v_{i} k$ (in which $k=|\mathbf{k}|$ and $v_{i}$ represents the phase velocity) representing the circular frequency, and the corresponding eigenvector $\mathbf{a}_{i}=\left(a_{i x}, a_{i y}, a_{i z}\right)$ represents the normalized polarization vector for the given mode. An alternative form of the above decomposition yields the following equation:

$$
\mathbf{\Gamma}=\mathbf{Q} \mathbf{\Lambda} \mathbf{Q}^{T}
$$

with

$$
\begin{gathered}
\boldsymbol{\Lambda}=\left(\begin{array}{ccc}
\lambda_{1}^{2} & 0 & 0 \\
0 & \lambda_{2}^{2} & 0 \\
0 & 0 & \lambda_{3}^{2}
\end{array}\right), \\
\mathbf{Q}=\left(\begin{array}{lll}
a_{1 x} & a_{2 x} & a_{3 x} \\
a_{1 y} & a_{2 y} & a_{3 y} \\
a_{1 z} & a_{2 z} & a_{3 z}
\end{array}\right) .
\end{gathered}
$$

Note that $\mathbf{Q}$ is an orthogonal matrix; i.e., $\mathbf{Q}^{-1}=\mathbf{Q}^{T}$.

The eigenvalues represent the frequencies and satisfy the condition that is given by the following equation:

$$
\lambda_{i}=v_{i} k \leq 2 \pi f_{\max },
$$

where $f_{\max }$ is the maximum frequency of the source. Therefore, we suggest to filter out the high-wavenumber components in the wavefields beyond $2 \pi f_{\max } / v_{\min }$ ( $v_{\min }$ is the minimum phase velocity in the computational model) caused by the numerical errors to enhance numerical stability.
According to above eigendecomposition, we apply the $k$-space adjustment to our pseudospectral scheme by modifying the eigenvalues of the Christoffel matrix for the anisotropic elastic wave equation (see Appendix C), i.e.,

$$
\overline{\boldsymbol{\Lambda}}=\left(\begin{array}{ccc}
\lambda_{1}^{2} \operatorname{sinc}^{2}\left(\lambda_{1} \Delta t / 2\right) & 0 & 0 \\
0 & \lambda_{2}^{2} \operatorname{sinc}^{2}\left(\lambda_{2} \Delta t / 2\right) & 0 \\
0 & 0 & \lambda_{3}^{2} \operatorname{sinc}^{2}\left(\lambda_{3} \Delta t / 2\right)
\end{array}\right) .
$$

Thus, this adjustment inserts a modified Christoffel matrix, i.e., $\overline{\mathbf{\Gamma}}=\mathbf{Q} \overline{\mathbf{\Lambda}} \mathbf{Q}^{T}$, into the original pseudospectral formula on the basis of equations 6 and 8 . Note that the $k$-space adjustment to the pseudospectral solution has been widely used in acoustic and ultrasound (Bojarski, 1982; Tabei et al., 2002) and elastic isotropic wavefield simulation (Liu, 1995; Firouzi et al., 2012).

\section{PROPAGATING DECOUPLED ELASTIC WAVEFIELDS}

The above pseudospectral operators propagate the elastic wavefields as a superposition of $\mathrm{qP}$ - and $\mathrm{qS}$-wave modes. To obtain
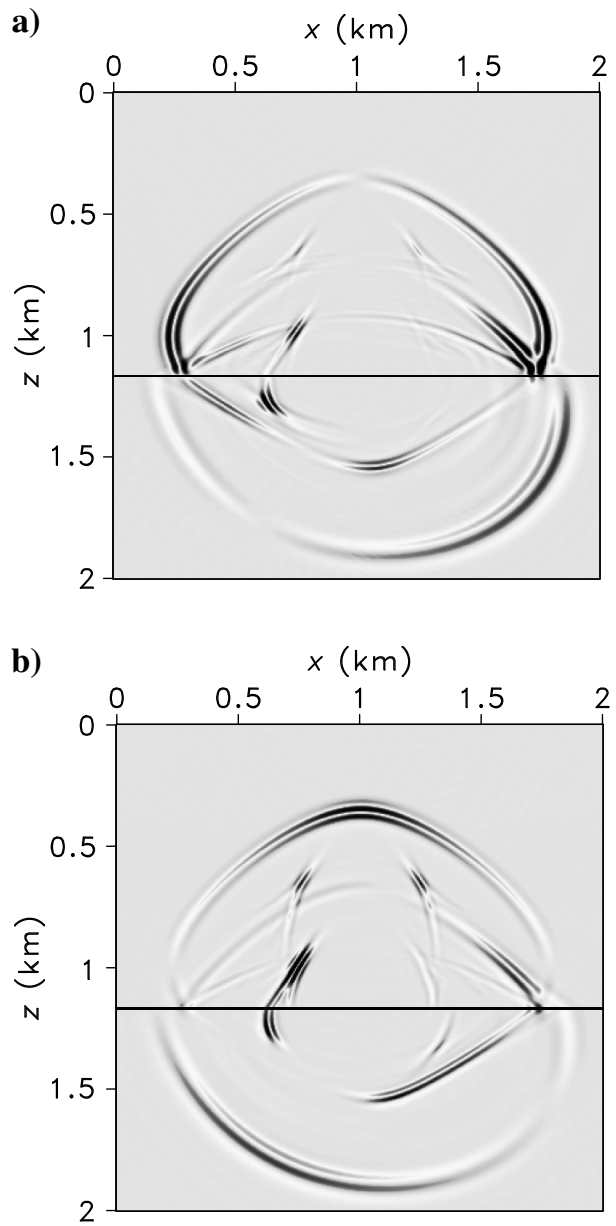

Figure 1. Horizontal (a) and vertical (b) components of the elastic wavefields at the time of $0.3 \mathrm{~s}$ synthesized by solving the secondorder elastic wave equation with $\Delta x=\Delta z=5 \mathrm{~m}$ and $\Delta t=0.5 \mathrm{~ms}$. 
physically interpretable results for seismic imaging and waveform inversion, wave mode decoupling is required during wavefield extrapolation. The key concept of mode decoupling is based on polarization. In a general anisotropic medium, the $\mathrm{qP}$ and $\mathrm{qS}$ modes do not polarize parallel and perpendicular to the wave vectors. Moreover, unlike the well-behaved $\mathrm{qP}$ mode, the two qS modes do not consistently polarize as a function of the propagation direction (or wavenumber) and thus cannot be designated as $\mathrm{SV}$ and $\mathrm{SH}$ waves, except in isotropic and TI media (Winterstein, 1990; Crampin, 1991). Even for a TI medium, it is a challenge to find the right solution of the shear singularity problem and obtain two completely separated S-modes with correct amplitudes (Yan and Sava, 2011; Cheng and Fomel, 2014). Therefore, we restrict to extrapolate the decoupled $\mathrm{qP}$ - and $\mathrm{qS}$-wave modes in this paper.
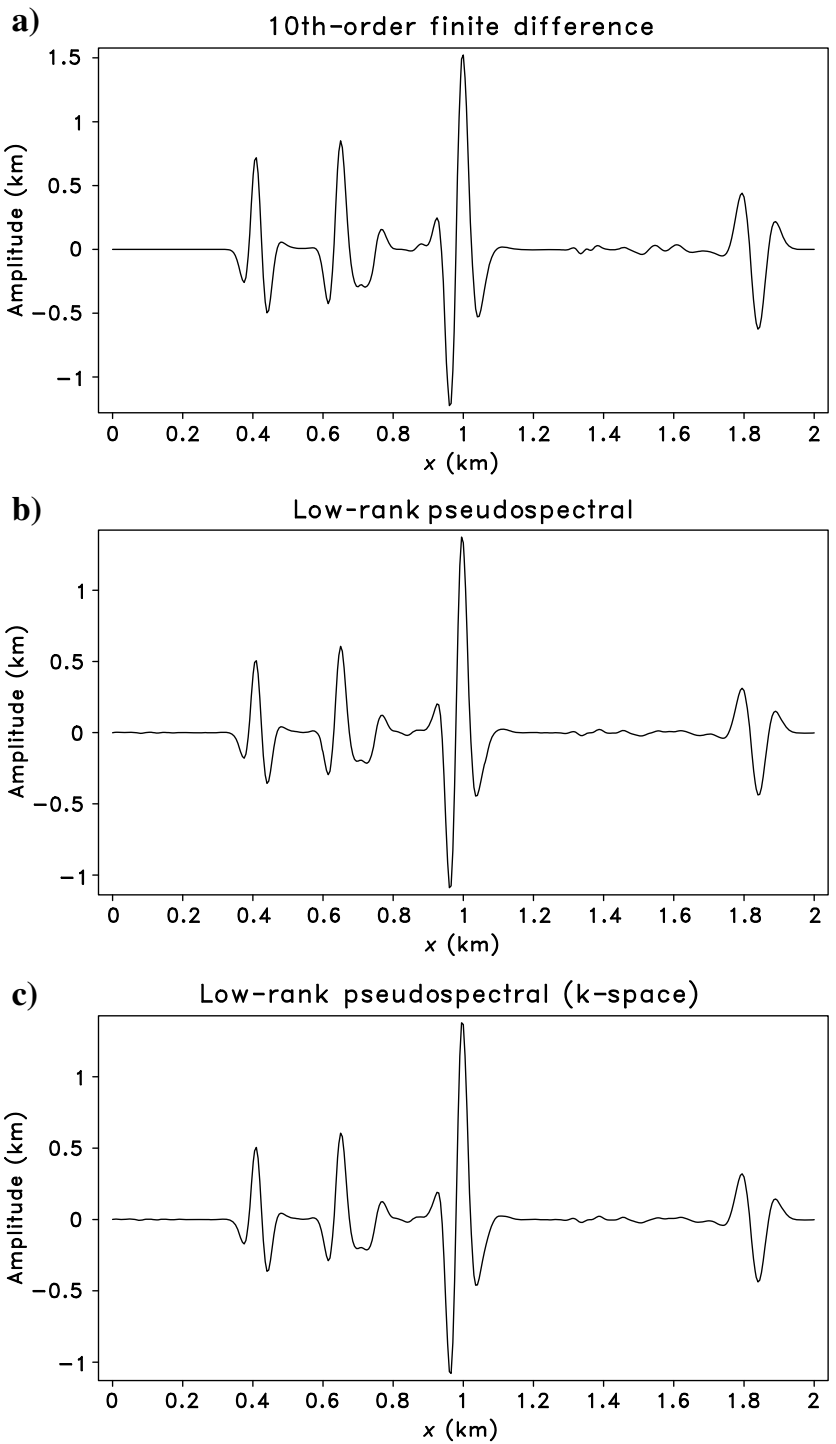

Figure 2. Vertical slices through the vertical components of the synthetic elastic wavefields at $x=0.75 \mathrm{~km}$ : (a) 10 th-order FD, (b) lowrank pseudospectral, and (c) low-rank pseudospectral using the $k$-space adjustment.

\section{Vector decomposition of the elastic wave modes}

According to Zhang and McMechan (2010), one can decompose $\mathrm{qP}$ and $\mathrm{qS}$ modes in the elastic wavefields for a homogeneous anisotropic medium using the following equation:

$$
u_{i}^{(m)}(\mathbf{k})=\mathbf{D}_{i j}^{(m)}(\mathbf{k}) \tilde{u}_{j}(\mathbf{k})
$$

where $m=\{\mathrm{qP}, \mathrm{qS}\}, i, j=\{x, y, z\}$, and the decomposition operators satisfy

$$
\begin{aligned}
& \mathbf{D}_{x x}^{(\mathrm{qP})}(\mathbf{k})=a_{x}^{2}(\mathbf{k}), \\
& \mathbf{D}_{y y}^{(\mathrm{qP})}(\mathbf{k})=a_{y}^{2}(\mathbf{k}), \\
& \mathbf{D}_{z z}^{(\mathrm{qP})}(\mathbf{k})=a_{z}^{2}(\mathbf{k}), \\
& \mathbf{D}_{x y}^{(\mathrm{qP})}(\mathbf{k})=a_{x}(\mathbf{k}) a_{y}(\mathbf{k}), \\
& \mathbf{D}_{x z}^{(\mathrm{qP})}(\mathbf{k})=a_{x}(\mathbf{k}) a_{z}(\mathbf{k}), \\
& \mathbf{D}_{y z}^{(\mathrm{qP})}(\mathbf{k})=a_{y}(\mathbf{k}) a_{z}(\mathbf{k}),
\end{aligned}
$$

and

$$
\begin{array}{ll}
\mathbf{D}_{x x}^{(\mathrm{qS})}(\mathbf{k})=a_{y}^{2}(\mathbf{k})+a_{z}^{2}(\mathbf{k}), & \mathbf{D}_{y y}^{(\mathrm{qS})}(\mathbf{k})=a_{x}^{2}(\mathbf{k})+a_{z}^{2}(\mathbf{k}), \\
\mathbf{D}_{z z}^{(\mathrm{qS})}(\mathbf{k})=a_{x}^{2}(\mathbf{k})+a_{y}^{2}(\mathbf{k}), & \mathbf{D}_{x y}^{(\mathrm{qS})}(\mathbf{k})=-a_{x}(\mathbf{k}) a_{y}(\mathbf{k}), \\
\mathbf{D}_{x z}^{(\mathrm{qS})}(\mathbf{k})=-a_{x}(\mathbf{k}) a_{z}(\mathbf{k}), & \mathbf{D}_{y z}^{(\mathrm{qS})}(\mathbf{k})=-a_{y}(\mathbf{k}) a_{z}(\mathbf{k}),
\end{array}
$$

where $a_{x}(\mathbf{k}), a_{y}(\mathbf{k})$, and $a_{z}(\mathbf{k})$ respectively represent the $x$-, $y$-, and $z$-components of the normalized polarization vector of the qP-wave.

As demonstrated by Cheng and Fomel (2014), one can decompose the wave modes in a heterogeneous anisotropic medium using the following mixed-domain integral operations:

$$
\begin{aligned}
& u_{x}^{(m)}(\mathbf{x})=\int e^{i \mathbf{k x}} \mathbf{D}_{x x}^{(m)}(\mathbf{x}, \mathbf{k}) \tilde{u}_{x}(\mathbf{k}) d \mathbf{k} \\
& +\int e^{i \mathbf{k x}} \mathbf{D}_{x y}^{(m)}(\mathbf{x}, \mathbf{k}) \tilde{u}_{y}(\mathbf{k}) d \mathbf{k} \\
& +\int e^{i \mathbf{k x}} \mathbf{D}_{x z}^{(m)}(\mathbf{x}, \mathbf{k}) \tilde{u}_{z}(\mathbf{k}) d \mathbf{k}, \\
& u_{y}^{(m)}(\mathbf{x})=\int e^{i \mathbf{k x}} \mathbf{D}_{x y}^{(m)}(\mathbf{x}, \mathbf{k}) \tilde{u}_{x}(\mathbf{k}) d \mathbf{k} \\
& +\int e^{i \mathbf{k x}} \mathbf{D}_{y y}^{(m)}(\mathbf{x}, \mathbf{k}) \tilde{u}_{y}(\mathbf{k}) d \mathbf{k} \\
& +\int e^{i \mathbf{k x}} \mathbf{D}_{y z}^{(m)}(\mathbf{x}, \mathbf{k}) \tilde{u}_{z}(\mathbf{k}) d \mathbf{k}, \\
& u_{z}^{(m)}(\mathbf{x})=\int e^{i \mathbf{k x}} \mathbf{D}_{x z}^{(m)}(\mathbf{x}, \mathbf{k}) \tilde{u}_{x}(\mathbf{k}) d \mathbf{k} \\
& +\int e^{i \mathbf{k x}} \mathbf{D}_{y z}^{(m)}(\mathbf{x}, \mathbf{k}) \tilde{u}_{y}(\mathbf{k}) d \mathbf{k} \\
& +\int e^{i \mathbf{k x}} \mathbf{D}_{z z}^{(m)}(\mathbf{x}, \mathbf{k}) \tilde{u}_{z}(\mathbf{k}) d \mathbf{k} .
\end{aligned}
$$




\section{Extrapolating the decoupled elastic waves}

For heterogeneous anisotropic media, the wavefield propagator (equation 6) and the vector decomposition operators (equation 18) are both in the general form of FIOs. Naturally, we merge them to

a)

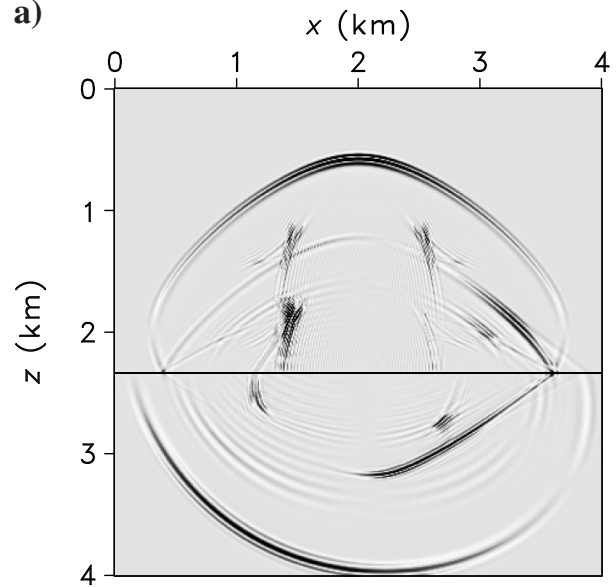

b)

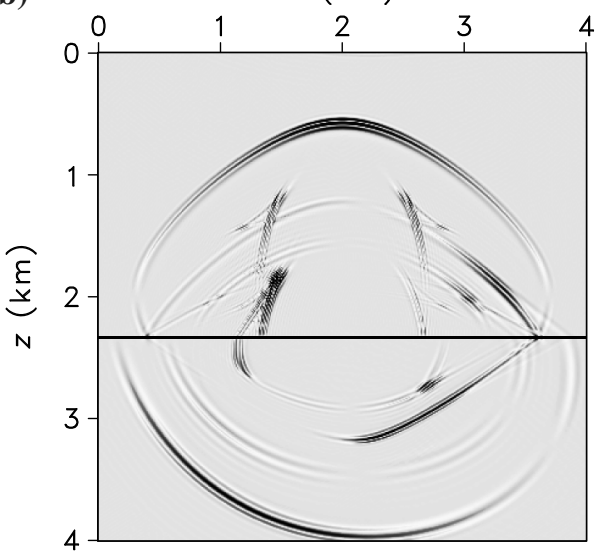

c)

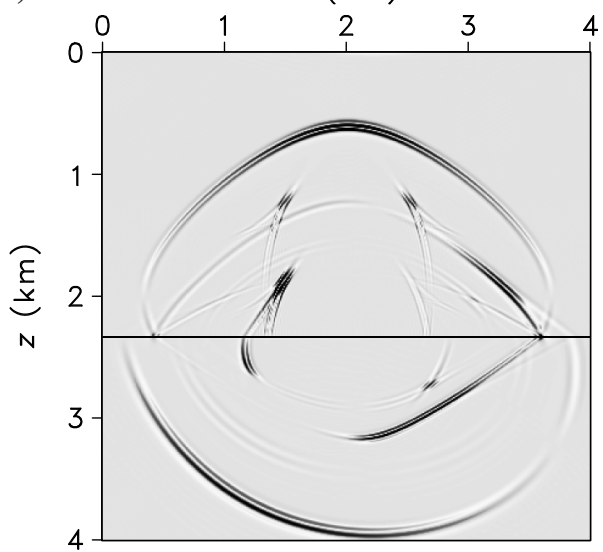

Figure 3. Vertical components of the elastic wavefields at the time of $0.6 \mathrm{~s}$ synthesized using three schemes with the same spatial sampling $\Delta x=\Delta z=10 \mathrm{~m}$ : (a) 10th-order FD, (b) low-rank pseudospectral with $\Delta t=1.5 \mathrm{~ms}$, and (c) low-rank pseudospectral solution using the $k$-space adjustment with $\Delta t=3 \mathrm{~ms}$. derive a new mixed-domain integral solution for extrapolating the decoupled elastic wavefields as follows:

$u_{x}^{(m)}(\mathbf{x}, t+\Delta t)=-u_{x}^{(m)}(\mathbf{x}, t-\Delta t)+\int e^{i \mathbf{k x}} \overline{\mathbf{W}}_{x x}^{(m)}(\mathbf{x}, \mathbf{k}) \tilde{u}_{x}(\mathbf{k}, t) d \mathbf{k}$

$+\int e^{i \mathbf{k} \mathbf{x}} \overline{\mathbf{W}}_{x y}^{(m)}(\mathbf{x}, \mathbf{k}) \tilde{u}_{y}(\mathbf{k}, t) d \mathbf{k}+\int e^{i \mathbf{k} \mathbf{x}} \overline{\mathbf{W}}_{x z}^{(m)}(\mathbf{x}, \mathbf{k}) \tilde{u}_{z}(\mathbf{k}, t) d \mathbf{k}$,

$u_{y}^{(m)}(\mathbf{x}, t+\Delta t)=-u_{y}^{(m)}(\mathbf{x}, t-\Delta t)+\int e^{i \mathbf{k x}} \overline{\mathbf{W}}_{y x}^{(m)}(\mathbf{x}, \mathbf{k}) \tilde{u}_{x}(\mathbf{k}, t) d \mathbf{k}$

$+\int e^{i \mathbf{k x}} \overline{\mathbf{W}}_{y y}^{(m)}(\mathbf{x}, \mathbf{k}) \tilde{u}_{y}(\mathbf{k}, t) d \mathbf{k}+\int e^{i \mathbf{k x}} \overline{\mathbf{W}}_{y z}^{(m)}(\mathbf{x}, \mathbf{k}) \tilde{u}_{z}(\mathbf{k}, t) d \mathbf{k}$,

$u_{z}^{(m)}(\mathbf{x}, t+\Delta t)=-u_{z}^{(m)}(\mathbf{x}, t-\Delta t)+\int e^{i \mathbf{k x}} \overline{\mathbf{W}}_{z x}^{(m)}(\mathbf{x}, \mathbf{k}) \tilde{u}_{x}(\mathbf{k}, t) d \mathbf{k}$

$+\int e^{i \mathbf{k x}} \overline{\mathbf{W}}_{z y}^{(m)}(\mathbf{x}, \mathbf{k}) \tilde{u}_{y}(\mathbf{k}, t) d \mathbf{k}+\int e^{i \mathbf{k x}} \overline{\mathbf{W}}_{z z}^{(m)}(\mathbf{x}, \mathbf{k}) \tilde{u}_{z}(\mathbf{k}, t) d \mathbf{k}$,
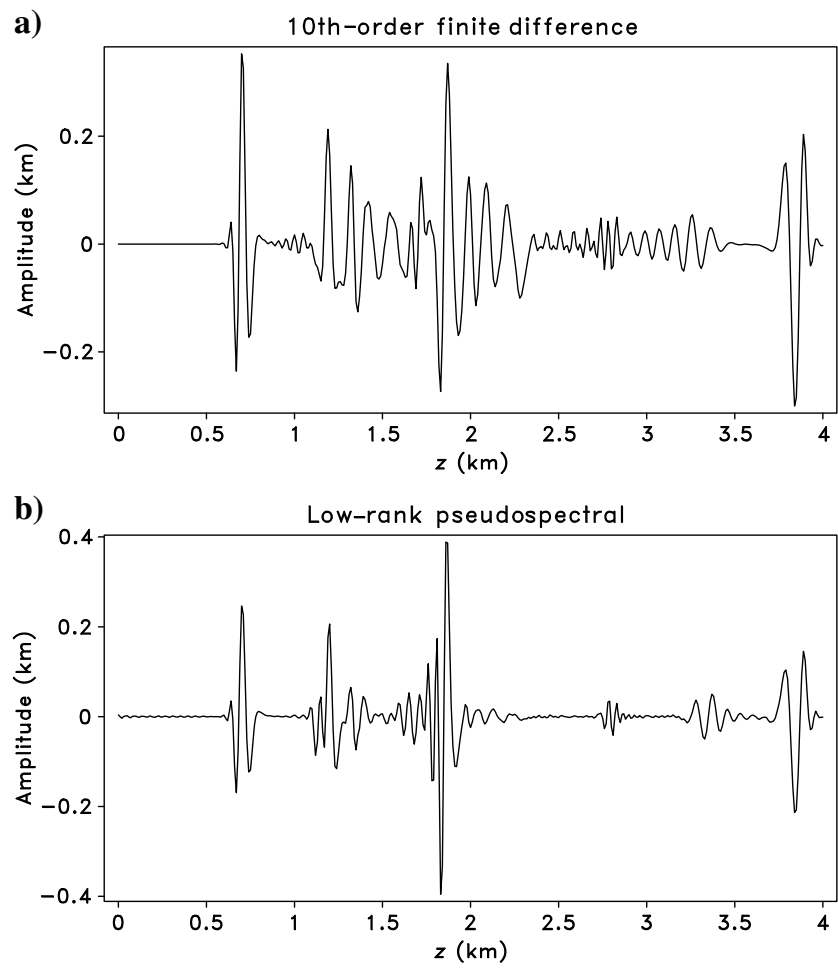

c) Low-rank pseudospectral (k-space)

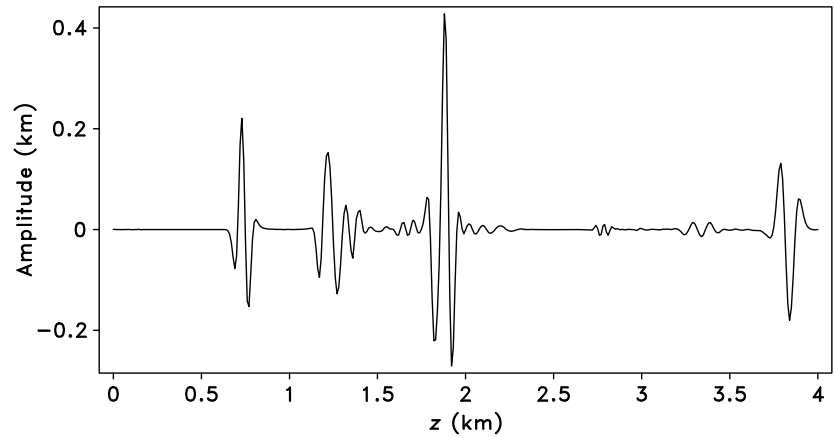

Figure 4. Vertical slices through the vertical components at $x=1.5 \mathrm{~km}$ in Figure 3: (a) 10th-order FD, (b) low-rank pseudospectral, and (c) low-rank pseudospectral using the $k$-space adjustment. 
with the propagation matrices for the decoupled wave modes given as

$$
\overline{\mathbf{W}}_{i j}^{(m)}(\mathbf{x}, \mathbf{k})=\mathbf{D}_{k i}^{(m)}(\mathbf{x}, \mathbf{k}) \mathbf{W}_{k j}(\mathbf{x}, \mathbf{k}),
$$

where $\mathbf{W}_{k j}(\mathbf{x}, \mathbf{k})$ is defined by the spatially varying Christoffel matrix and the length of time step, namely,

$$
\begin{aligned}
& \mathbf{W}_{k k}(\mathbf{x}, \mathbf{k})=2-\Delta t^{2} \Gamma_{k k}(\mathbf{x}, \mathbf{k}), \\
& \mathbf{W}_{k j}(\mathbf{x}, \mathbf{k})=-\Delta t^{2} \Gamma_{k j}(\mathbf{x}, \mathbf{k})
\end{aligned}
$$

The extended formulation of equation 20 is given in Appendix B. Note that symmetry properties exist: $\mathbf{D}_{k i}^{(m)}=\mathbf{D}_{i k}^{(m)}$ and $\mathbf{W}_{k j}=\mathbf{W}_{j k}$, and the modified Christoffel matrix will be used if the $k$-space adjustment is applied for the pseudospectral solutions.
To derive the time extrapolation of the decomposed wavefields using equation 19, we must update the total elastic wavefields by superposing $\mathrm{qP}$ - and $\mathrm{qS}$-waves at each time step using the following equation:

$$
\begin{aligned}
& u_{x}(\mathbf{x})=u_{x}^{(\mathrm{qP})}(\mathbf{x})+u_{x}^{(\mathrm{qS})}(\mathbf{x}), \\
& u_{y}(\mathbf{x})=u_{y}^{(\mathrm{qP})}(\mathbf{x})+u_{y}^{(\mathrm{qS})}(\mathbf{x}), \\
& u_{z}(\mathbf{x})=u_{z}^{(\mathrm{qP})}(\mathbf{x})+u_{z}^{(\mathrm{qS})}(\mathbf{x}) .
\end{aligned}
$$

Thus, equations 19-22 compose the spectral-like operators to simultaneously extrapolate and decouple the elastic wavefields for 3D anisotropic media. The computation complexity of the straight-
Figure 5. Elastic wavefields at the time of $0.6 \mathrm{~s}$ synthesized by using low-rank pseudospectral solution of the displacement wave equation followed with low-rank vector decomposition: (a) $x$ - and (b) $z$-components of the displacement wavefields, (c) $x$ - and (d) $z$-components of the qP wavefields, and (e) $x$ - and (f) $z$-components of the qSV wavefields. a)

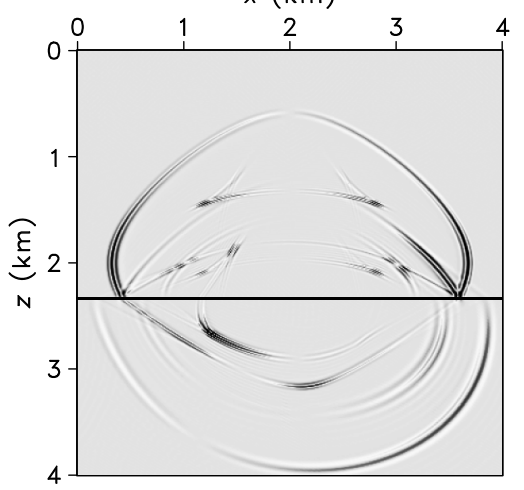

c)

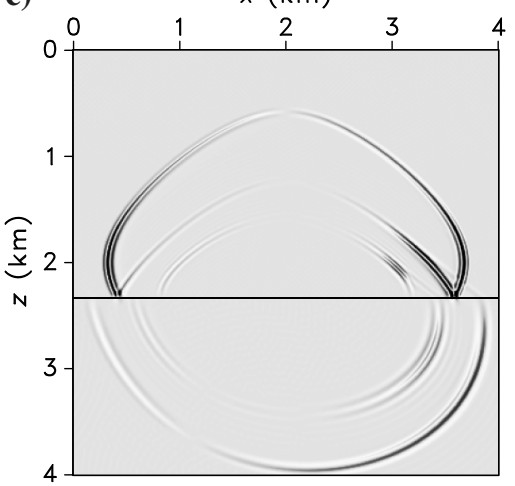

e)

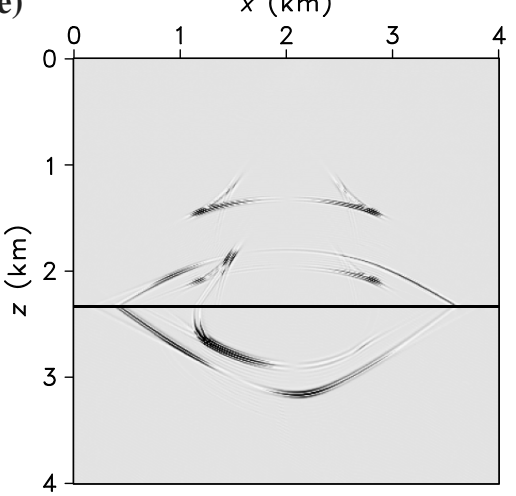

b) $\quad x(\mathrm{~km})$

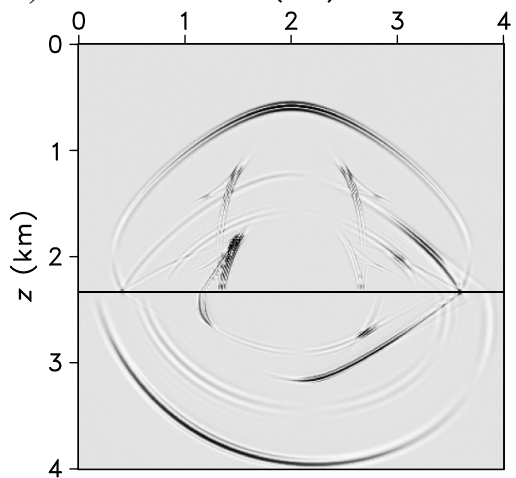

d)

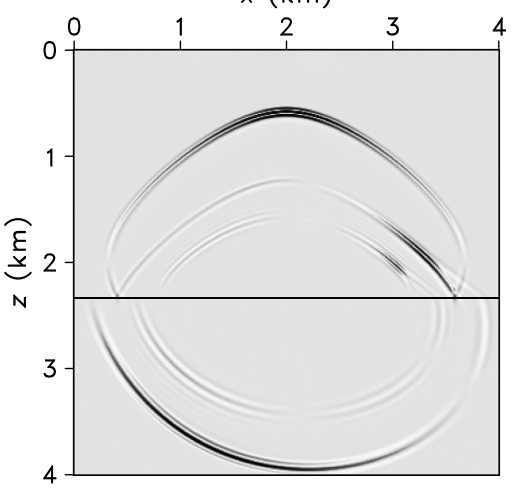

f)

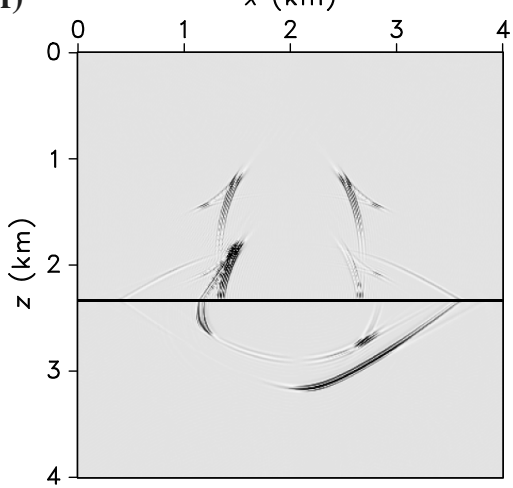


forward implementation of the integral operators in equations 8 and 19 is $O\left(N_{x}^{2}\right)$, which is prohibitively expensive when the size of model $N_{x}$ is large.

To tackle strong heterogeneity due to fast varying stiffness coefficients, we suggest splitting the displacement equation into the displacement-stress equation and then solving it using the staggered-grid pseudospectral scheme (Ozdenvar and McMechan, 1996; Carcione, 1999; Bale, 2003). Note that when using staggered grids, the operators to extrapolate the decoupled wave modes must be modified to account for the shifts in medium properties and fields variables. We will investigate this issue in future work.

\section{FAST ALGORITHM USING LOW-RANK DECOMPOSITION}

As proposed by Cheng and Fomel (2014), low-rank decomposition of the mixed-domain matrix $d(\mathbf{x}, \mathbf{k})$ in equation 18 yields a very efficient algorithm for mode decoupling in heterogeneous anisotropic media. We find that the same strategy works for numerical implementations of above pseudospectral operators for elastic wave propagation

For example, the mixed-domain matrix, i.e., $\mathbf{W}(\mathbf{x}, \mathbf{k})$ or $\overline{\mathbf{W}}(\mathbf{x}, \mathbf{k})$ in the FIOs, can be approximated by the following separated representation (Fomel et al., 2013): a)

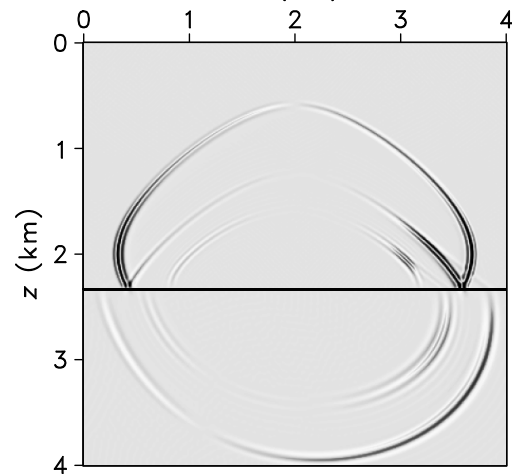

c)

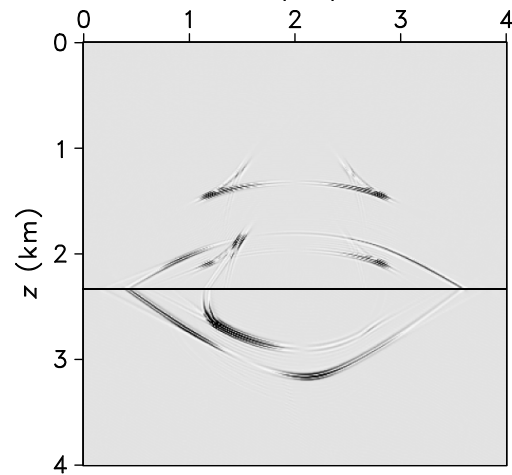

e)

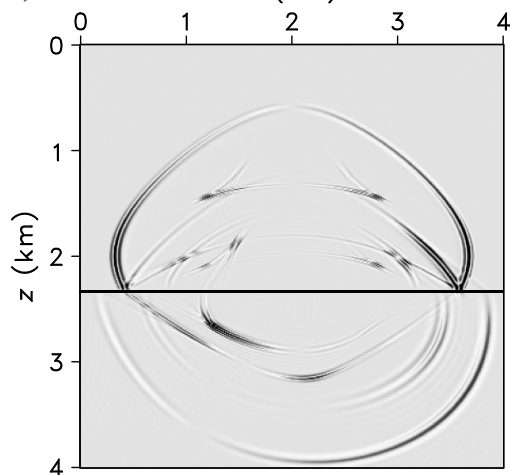

b)

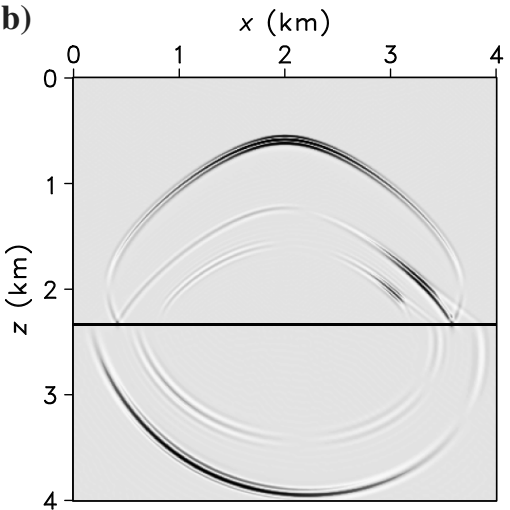

d)

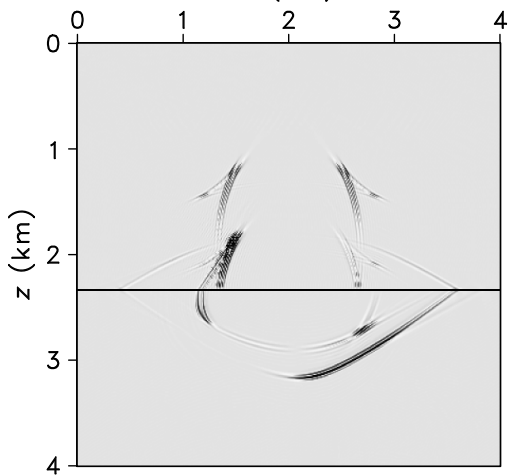

f)

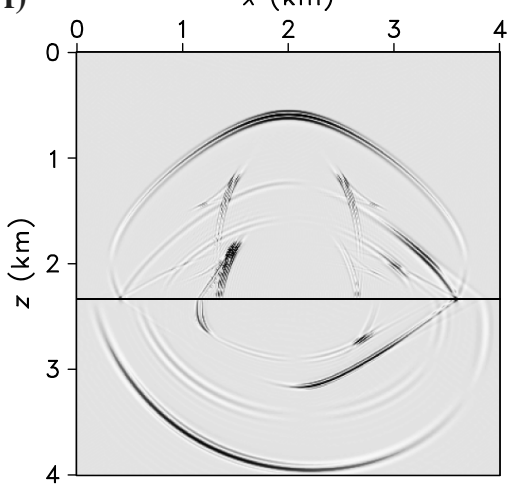

Figure 6. Elastic wavefields at the time of $0.6 \mathrm{~s}$ synthesized by using low-rank pseudospectral operators for extrapolating and decomposing the elastic waves simultaneously: (a) $x$ - and (b) $z$-components of the qP-wave displacement wavefields, (c) $x$ - and (d) $z$-components of the qSV-wave displacement wavefields, and (e) $x$ - and (f) $z$-components of the total elastic wavefields. 


$$
W(\mathbf{x}, \mathbf{k}) \approx \sum_{m=1}^{M} \sum_{n=1}^{N} B\left(\mathbf{x}, \mathbf{k}_{m}\right) A_{m n} C\left(\mathbf{x}_{n}, \mathbf{k}\right),
$$

where $B\left(\mathbf{x}, \mathbf{k}_{m}\right)$ is a mixed-domain matrix with reduced wavenumber dimension $M, C\left(\mathbf{x}_{n}, \mathbf{k}\right)$ is a mixed-domain matrix with reduced spatial dimension $N$, and $A_{m n}$ is an $M \times N$ matrix with $N$ and $M$ representing the rank of this decomposition. Physically, a separable low-rank approximation amounts to selecting a set of $N\left(N \ll N_{x}\right)$ representative spatial locations and $M\left(M \ll N_{x}\right)$ representative wavenumbers. Construction of the separated representation follows the method of Engquist and Ying (2009). The ranks $M$ and $N$ are dependent on the complexities (heterogeneity and anisotropy) of the medium and the estimate of the approximation accuracy to the mixed-domain matrices (in the numerical examples, we aim for the relative single-precision accuracy of $10^{-6}$ ). More explanations on low-rank decomposition are available in Fomel et al. (2013) and Cheng and Fomel (2014). As we observe, the ranks are generally very small for our applications. For homogeneous media, the ranks naturally reduce to one. If there is heterogeneity, the ranks increase to two for isotropic media but exceed two for anisotropic media. The $k$-space adjustment may slightly increase the ranks for the heterogeneous media.

Thus, the above low-rank approximation speeds up computation of the FIOs as follows:

$$
\begin{aligned}
& \int e^{i \mathbf{k x}} W(\mathbf{x}, \mathbf{k}) \tilde{u}_{j}(\mathbf{k}) d \mathbf{k} \\
& \approx \sum_{m=1}^{M} B\left(\mathbf{x}, \mathbf{k}_{m}\right)\left(\sum_{n=1}^{N} A_{m n}\left(\int e^{i \mathbf{k} \mathbf{x}} C\left(\mathbf{x}_{n}, \mathbf{k}\right) \tilde{u}_{j}(\mathbf{k}) d \mathbf{k}\right)\right)
\end{aligned}
$$
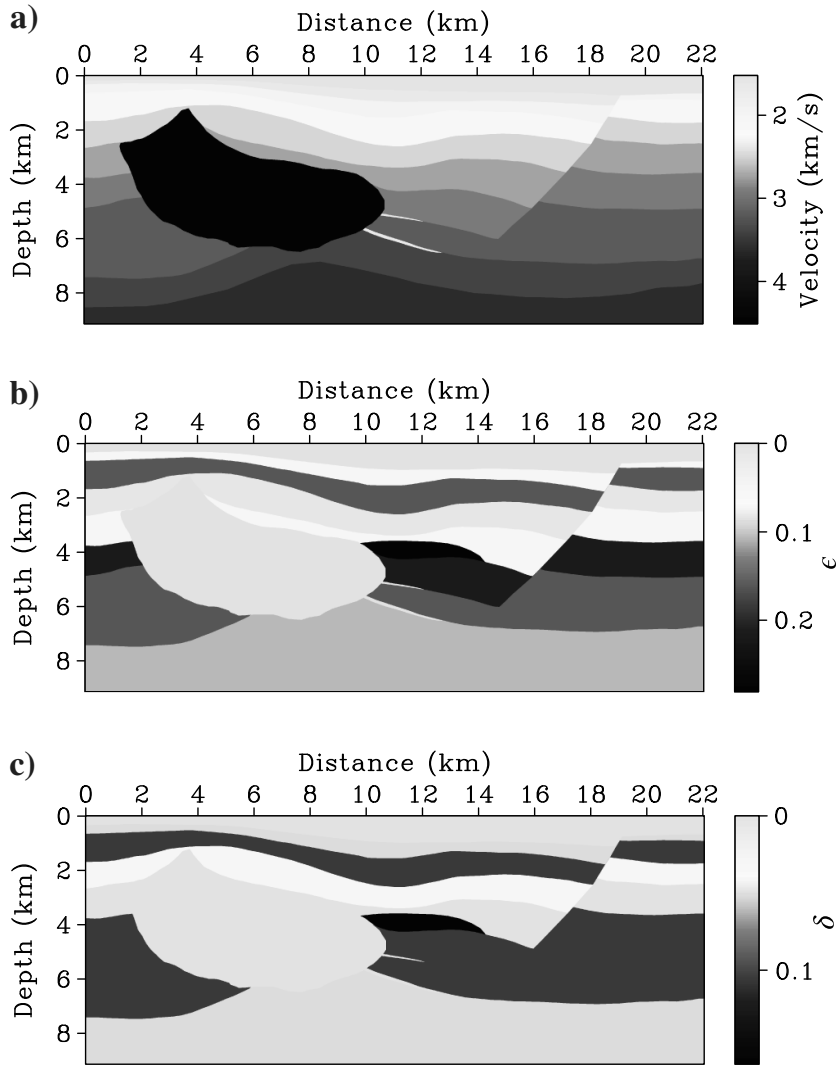

Figure 8. The SEG/Hess VTI model with parameters of (a) vertical $\mathrm{P}$-wave velocity, Thomsen coefficients (b) $\epsilon$, and (c) $\delta$.
Figure 7. Synthesized decomposed and total elastic wavefields for a orthorhombic model with a VTI overburden: qP (top), qS (mid), and total (bottom) elastic displacement fields (left, $x$-component; mid, $y$-component; and right, $z$-component).
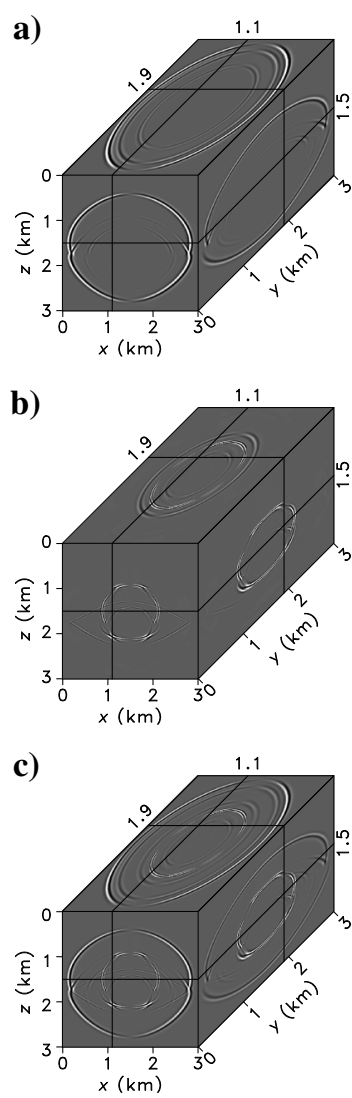
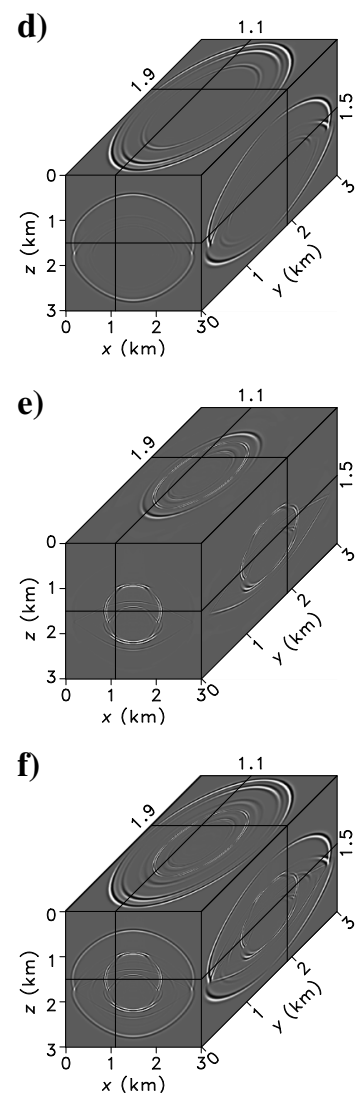
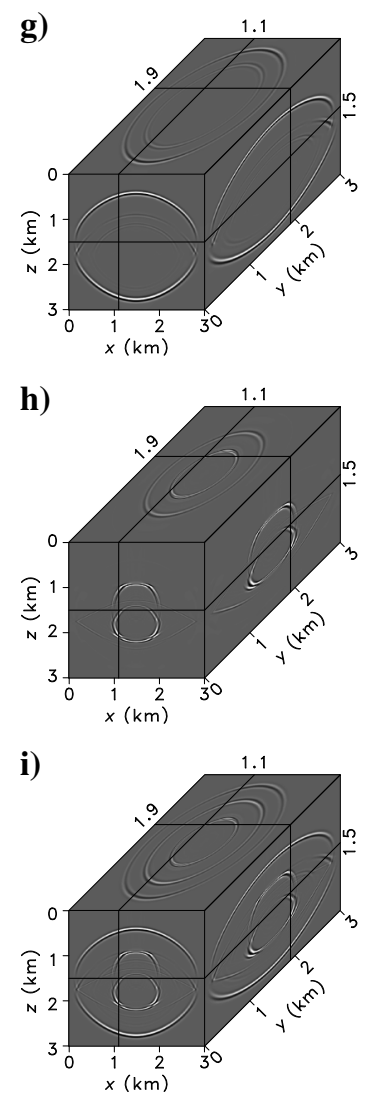
Evaluation of the last formula is effectively equivalent to applying $N$ inverse FFTs each time step. Accordingly, the computation complexity reduces to $O\left(N N_{x} \log N_{x}\right)$. In multiple-core implementations, the matrix operations in equation 24 are easy to parallelize.

\section{EXAMPLES}

We will first demonstrate the proposed approach on two-layer TI and orthorhombic models, and then on the complex SEG Hess VTI and BP 2007 TTI models, respectively.

\section{D two-layer VTI/TTI model}

The first example is on a 2D two-layer model, in which the first layer is a VTI medium with $V_{\mathrm{P} 0}=2500 \mathrm{~m} / \mathrm{s}, V_{\mathrm{S} 0}=1200 \mathrm{~m} / \mathrm{s}$, $\epsilon=0.2$, and $\delta=-0.2$, and the second layer is a tilted TI
(TTI) medium with $V_{\mathrm{P} 0}=3600 \mathrm{~m} / \mathrm{s}, V_{\mathrm{S} 0}=1800 \mathrm{~m} / \mathrm{s}, \epsilon=0.2$, $\delta=0.1$, and $\theta=30^{\circ}$. A point source is placed at the center of this model. First, we compare the synthetic elastic wavefields by solving the elastic displacement wave equation using the 10th-order explicit FD and low-rank pseudospectral schemes (with or without the $k$-space adjustment), respectively. Figure 1 shows the wavefield snapshots at the time of $0.3 \mathrm{~s}$ using the spatial sampling $\Delta x=\Delta z=$ $5 \mathrm{~m}$ and time step $\Delta t=0.5 \mathrm{~ms}$. Only the low-rank pseudospectral solutions with the $k$-space adjustment are displayed because the three schemes produce very similar results. The vertical slices through the $z$-components of the elastic wavefields show few differences among them (Figure 2). For the low-rank pseudospectral scheme, the ranks are all two for the decomposition of the mixeddomain matrices $\mathbf{W}_{x x}, \mathbf{W}_{z z}$, and $\mathbf{W}_{x z}$ in equation 21 , and the $k$-space adjustment does not change the ranks. It takes CPU times of 0.20 , 0.23 , and $0.23 \mathrm{~s}$ for them to finish the wavefield extrapolation of one a)

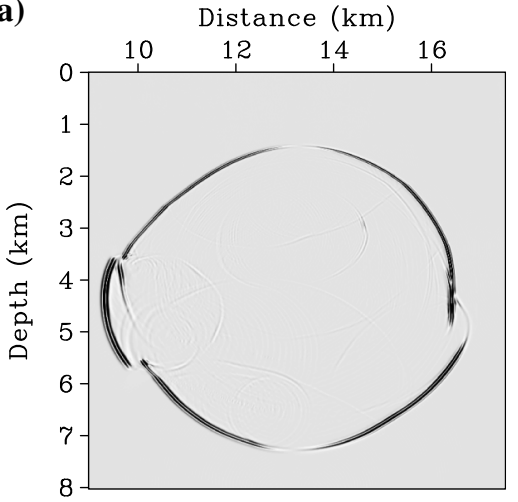

c)

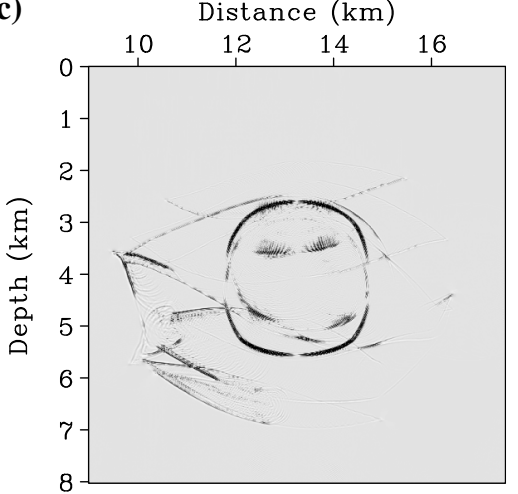

e)

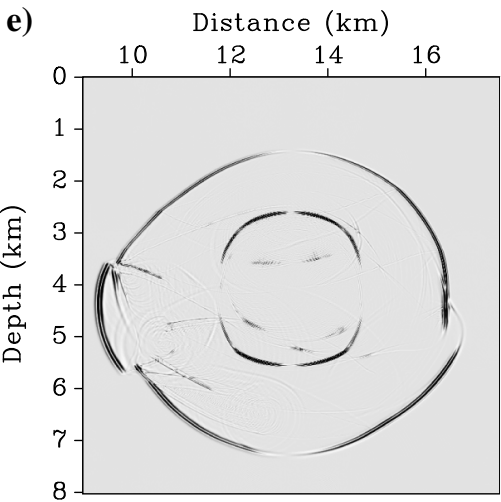

b)

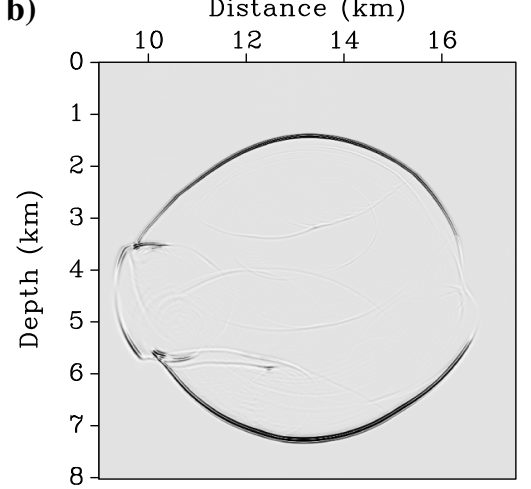

d)

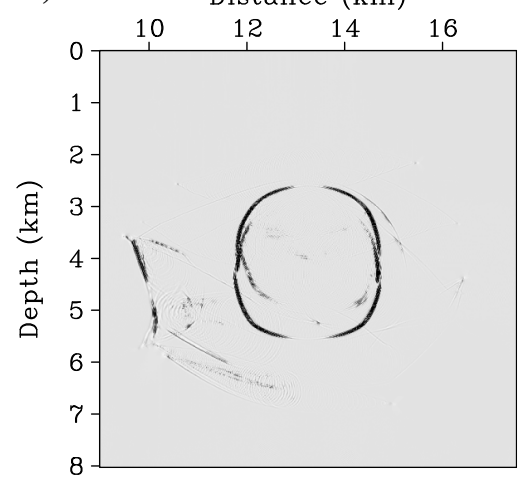

f)

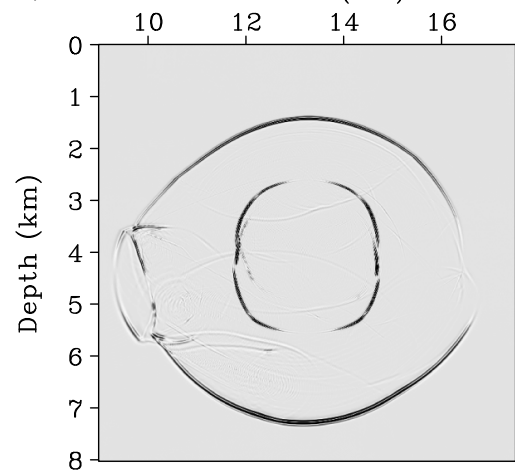

Figure 9. Synthesized decoupled and total displacement fields using the low-rank pseudospectral solution with the $k$-space adjustment that simultaneously extrapolate and decouple $\mathrm{qP}$ and $\mathrm{qSV}$ wavefields in SEG/Hess VTI model: (a) $x$ - and (b) $z$-components of qP-wavefields, (c) $x$ - and (d) $z$-components of qSV wavefields, and (e) $x$ and (f) $z$-components of the total displacement fields. 
time step. An additional 4.3 and $8.2 \mathrm{~s}$ have been used to finish the low-rank decomposition of the involved mixed-domain matrices before wavefield extrapolation. We observe the FD scheme is unstable if the time step is increased to $1.0 \mathrm{~ms}$ and the low-rank pseudospectral scheme is unstable if the time step is increased to $2.0 \mathrm{~ms}$ (with unchanged spatial sampling). However, the low-rank pseudospectral solution using the $k$-space adjustment produces acceptable results even the time step is increased to $3.0 \mathrm{~ms}$ and the maximum time exceeds $3 \mathrm{~s}$. Figures 3 and 4 compare the wavefield snapshots and the vertical slices at the time of $0.6 \mathrm{~s}$ using the three schemes with the increased spatial sampling (namely, $\Delta x=\Delta z=10 \mathrm{~m}$ ). The FD scheme tends to exhibit dispersion artifacts with the chosen model size and extrapolation step, whereas the low-rank pseudospectral scheme exhibits acceptable accuracy. The $k$-space adjustment permits larger time steps without reducing accuracy or introducing instability. For this example, it has produced the best results with less numerical dispersion. Thanks to the larger spatial and temporal sampling, the same CPU time is used for each scheme as in Figure 1. In addition, only the ranks for the low-rank decomposition of the matrix $w_{12}$ reduce to one when we change the tilt angle of the second layer to zero.

Second, we compare two approaches to get the decoupled elastic wavefields during time extrapolation. The first approach uses the low-rank pseudospectral algorithm to synthesize the elastic wavefields and then applies the low-rank vector decomposition algorithm (Cheng and Fomel, 2014) to get the vector $\mathrm{qP}$ and $\mathrm{qSV}$ wavefields (Figure 5). The second extrapolates the decoupled $\mathrm{qP}$ - and $\mathrm{qSV}$ wavefields using the proposed low-rank mixed-domain integral operations (Figure 6). Extrapolation steps of $\Delta x=\Delta z=10 \mathrm{~m}$ and $\Delta t=1.0 \mathrm{~ms}$ are used in this example. The ranks are still two for the involved low-rank decomposition of the propagation matrices defined in equation 20. The two approaches produce compa-
Figure 10. Elastic wavefield extrapolation using 10th-order FD scheme and low-rank vector decomposition in SEG/Hess VTI model: (a) $x$ - and (b) $z$ components of the synthetic elastic displacement wavefields at $1.1 \mathrm{~s}$, (c) $x$ - and (d) $z$-components of vector qP wavefields, and (e) $x$ - and (f) $z$-components of vector qSV wavefields. a)

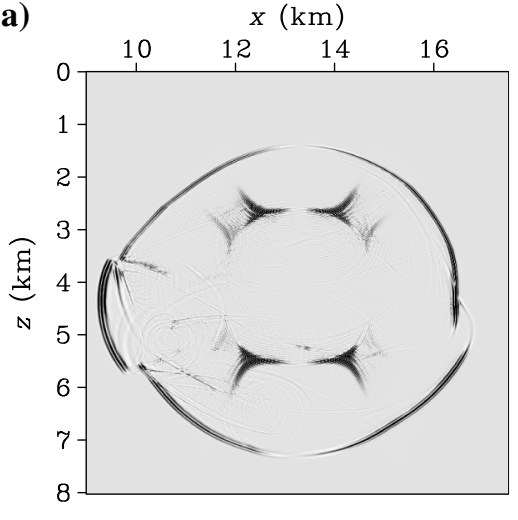

c)

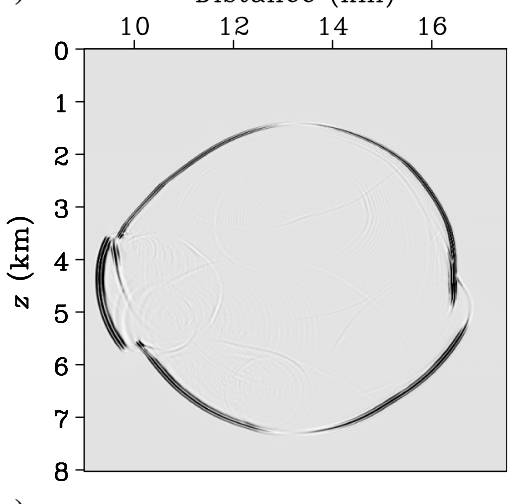

e)

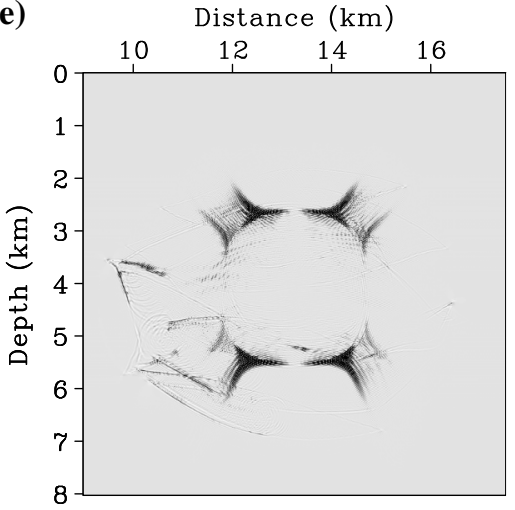

b)

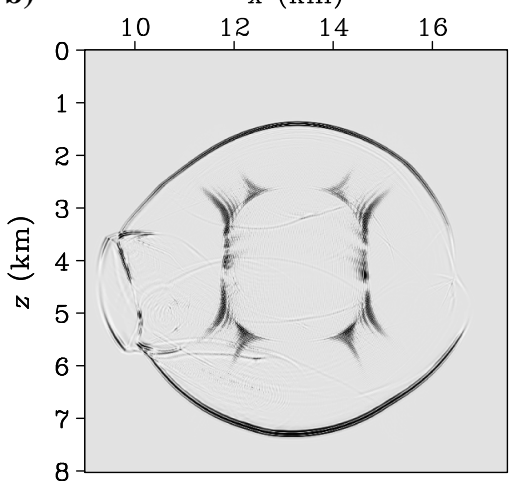

d)

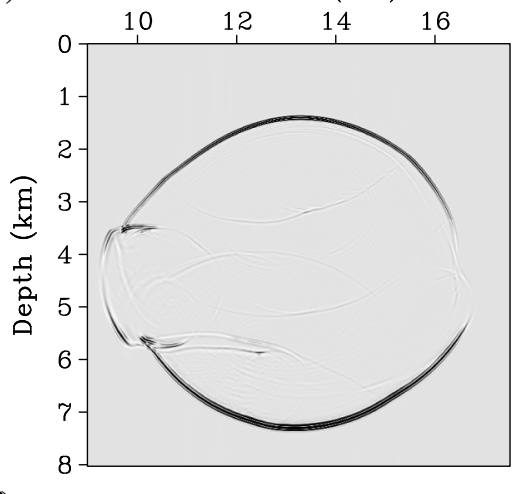

f)

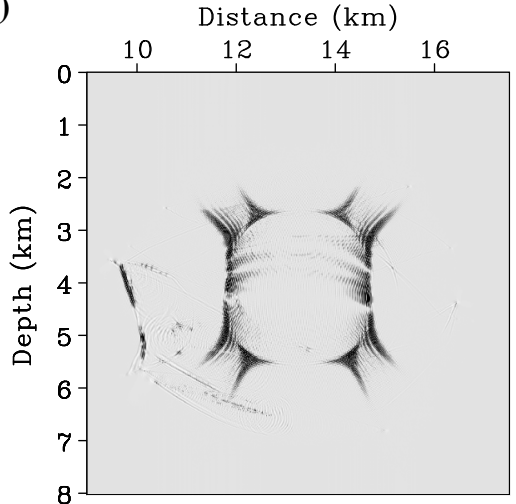


rable elastic wavefields, in which we can observe all transmitted and reflected waves including mode conversions. For one step of the time extrapolation, it takes the CPU time of $0.6 \mathrm{~ms}$ for the first approach and $0.5 \mathrm{~ms}$ for the second. This means that merging time extrapolation and vector decomposition into a unified Fourier integral framework provides a more efficient solution than operating them in sequence for anisotropic media thanks to the reduced number of forward and inverse FFTs.

\section{D two-layer VTI/orthorhombic model}

Figure 7 shows synthetic vector displacement fields using the proposed approach for a 3D two-layer model, with a horizontal reflector at $1.167 \mathrm{~km}$. The first layer is a VTI medium with $V_{\mathrm{P} 0}=2500 \mathrm{~m} / \mathrm{s}, V_{\mathrm{S} 0}=1400 \mathrm{~m} / \mathrm{s}, \epsilon=0.25, \delta=0.05$, and $\gamma=0.15$, and the second layer is an orthorhombic medium representing a vertically fractured TI formation (Schoenberg and Helbig, 1997; Tsvankin, 2001), which has the parameters $V_{\mathrm{P} 0}=3000 \mathrm{~m} / \mathrm{s}$, $V_{\mathrm{S} 0}=1600 \mathrm{~m} / \mathrm{s}, \epsilon_{1}=0.30, \epsilon_{2}=0.15, \delta_{1}=0.08, \delta_{2}=-0.05$, $\delta_{3}=-0.10, \gamma_{1}=0.20$, and $\gamma_{2}=0.05$. An exploration source is located at the center of the model. We achieve efficient simulation of dispersion-free 3D elastic wave propagation for the decoupled and total displacement fields. S-wave splitting can be observed in the qS-wavefields.

a)

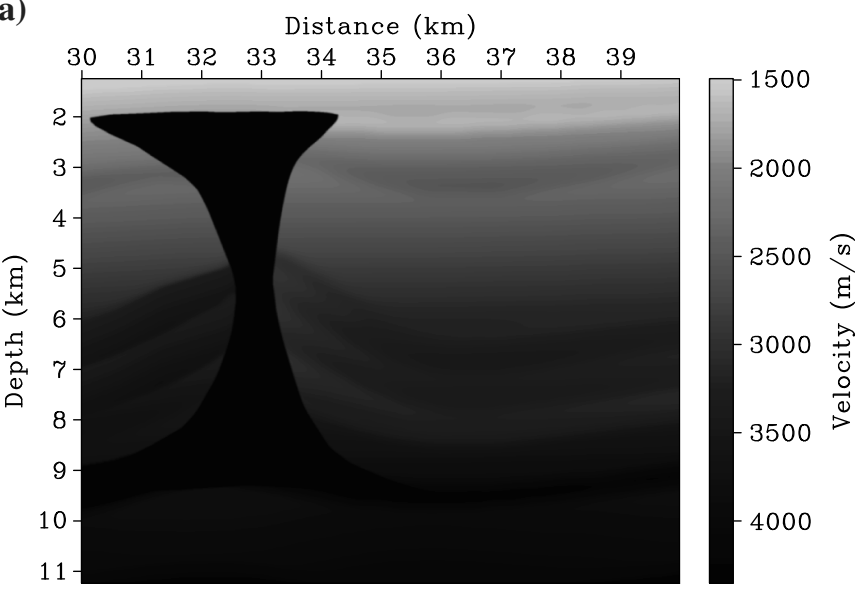

c)

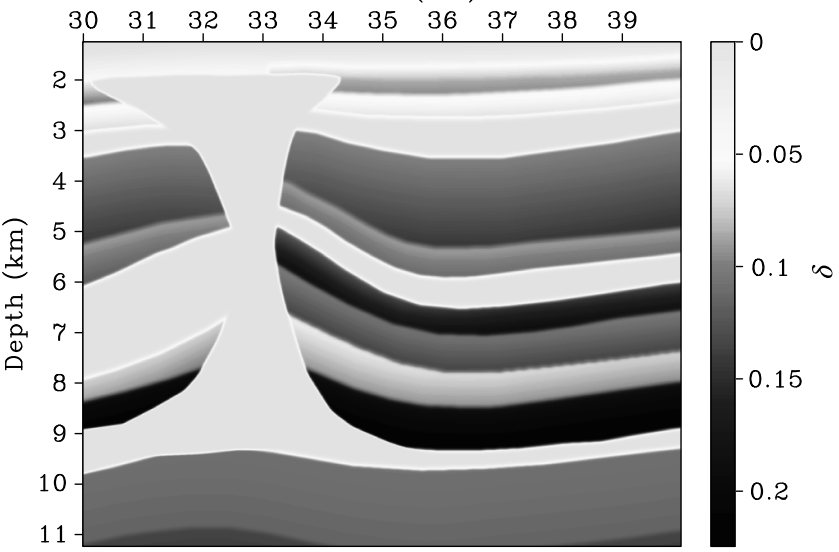

\section{SEG Hess VTI model}

Then, we demonstrate the approach in the 2D Hess VTI model (Figure 8). The vertical qS-wave velocity is set to equal half the vertical qP-wave velocity everywhere. A point source is placed at a location of $(13.264,4.023) \mathrm{km}$. For comparison, a spatial step length of $\Delta x=\Delta z=40.0 \mathrm{ft}$ and a time step of $\Delta t=1 \mathrm{~ms}$ are used in this example. Figure 9 displays the decoupled and total displacement fields synthesized by using the low-rank pseudospectral algorithm that simultaneously extrapolates the decoupled $\mathrm{qP}$ - and qSV-wave modes. The ranks $N, M$ are in $[8,10]$ for the low-rank decomposition of the involved matrices (the ranks reduce to $[1,3]$ if we only propagate the coupled elastic wavefields). The wavefield snapshots show that the proposed wave propagator honors the elastic effects such as mode conversion. It takes the CPU $111.6 \mathrm{~s}$ to decompose the mixeddomain matrices in advance, and it takes approximately $9397.7 \mathrm{~s}$ to extrapolate the decoupled wavefields to the maximum time of $1100 \mathrm{~ms}$. Figure 10 displays the total displacement fields synthesized by the 10th-order FD solution of the elastic wave equation and the decoupled qP- and qSV-wavefields using the low-rank vector decomposition for heterogeneous TI media (Cheng and Fomel, 2014). The ranks are in [6,7] for the decomposition of the involved mode decoupling matrices $d_{i j}$. The FD solution shows strong numerical dispersion of the $\mathrm{qSV}$ waves due to inadequate sampling because

b)

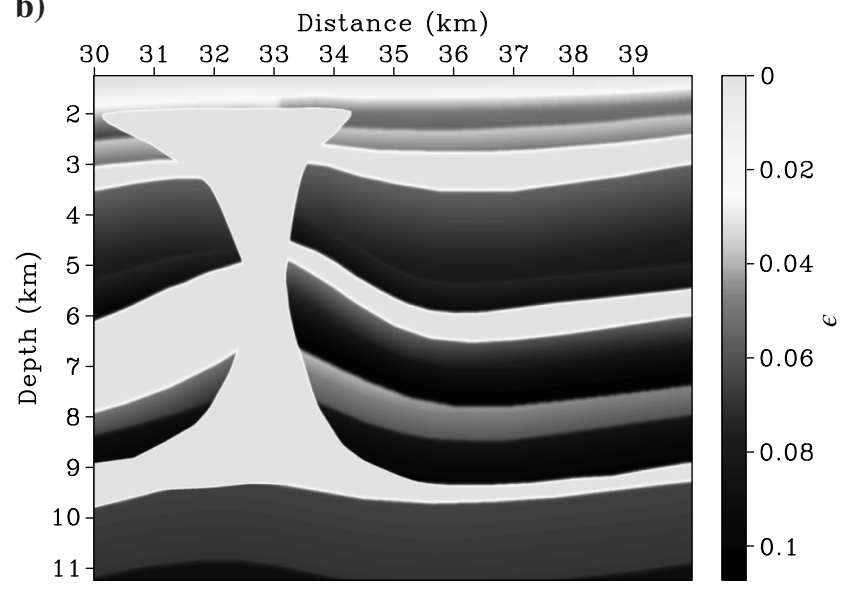

d)

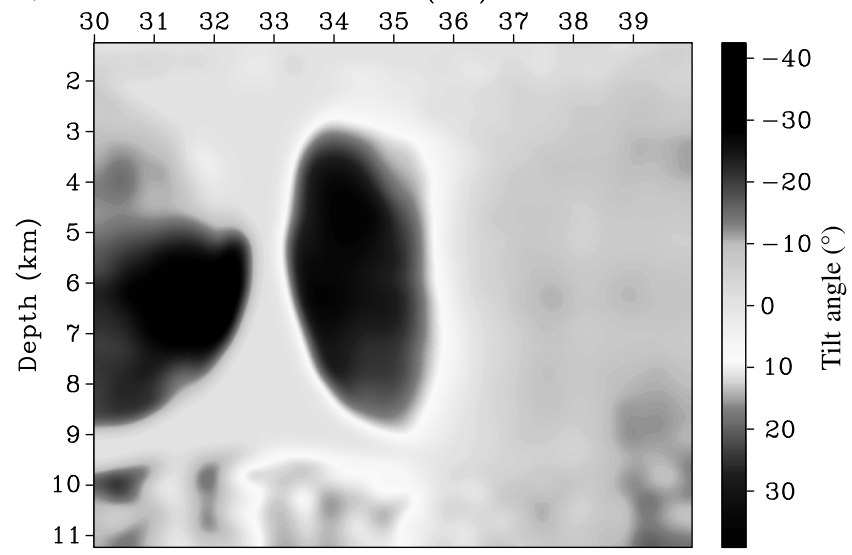

Figure 11. Partial of BP 2007 TTI model with parameters of (a) vertical P-wave velocity, Thomsen coefficients (b) $\epsilon$, (c) $\delta$, and (d) tilt angle $\theta$. 
the modeling of the qSV wave using the FD scheme demands a finer grid cell size. Except for the CPU time of $36.7 \mathrm{~s}$ to decompose the mixed-domain matrices for mode decoupling, it takes $568 \mathrm{~s}$ to extrapolate and $4690.3 \mathrm{~s}$ to decouple the elastic wavefields to get $\mathrm{qP}$ and qS wavefields for all the time steps. To achieve the same good quality as the low-rank pseudospectral solution in Figure 8, we decrease the spatial sampling to $\Delta x=\Delta z=20 \mathrm{ft}$ and the temporal sampling to $0.5 \mathrm{~ms}$. Except the CPU time of $133.8 \mathrm{~s}$ to decompose the mixed-domain matrices for mode decoupling, it takes $3922.8 \mathrm{~s}$ to extrapolate and $14,212 \mathrm{~s}$ to decouple the elastic wavefields to the maximum time. This means the low-rank pseudospectral scheme is more efficient to obtain decoupled elastic wavefields for TI media.

\section{BP 2007 TTI model}

The last example displays the application to the BP 2007 TTI model (Figure 11). The vertical qS-wave velocity is set to equal $60 \%$ of the vertical qP-wave velocity everywhere. Extrapolation steps of $\Delta x=\Delta z=12.5 \mathrm{~m}$ and $\Delta t=1 \mathrm{~ms}$ are used here. Because the principal axes of the medium are not aligned with the Cartesian axes, we have to apply the Bond transformation to get the stiffness matrix under the Cartesian system. Before wavefield extrapolation, separated representations of the mixed operator matrixes are constructed using the low-rank decomposition approach within the computational zone. For this complex model, the ranks are approximately 30 for the decomposition of the involved matrices. As shown in Figure 12, the approach describes very well the propagations of the decoupled $\mathrm{qP}$ and $\mathrm{qS}$ waves as well as the total displacement fields even for this complex TTI model. We can clearly observe the converted waves from the dipping salt flanks and other strong-contrast interfaces. And the $\mathrm{qP}$ and $\mathrm{qS}$ waves are free of numerical dispersion in the decoupled and total wavefields.
Figure 12. Synthesized decoupled and total displacement fields at the time of $1.2 \mathrm{~s}$ using the low-rank pseudospectral solution with the $k$-space adjustment that simultaneously extrapolate and decouple qP and qSV wavefields in the BP 2007 TI model: (a) $x$ - and (b) $z$-components of qP wavefields, (c) $x$ - and (d) $z$-components of qSV wavefields, and (e) $x$ - and (f) $z$-components of the total displacement fields.
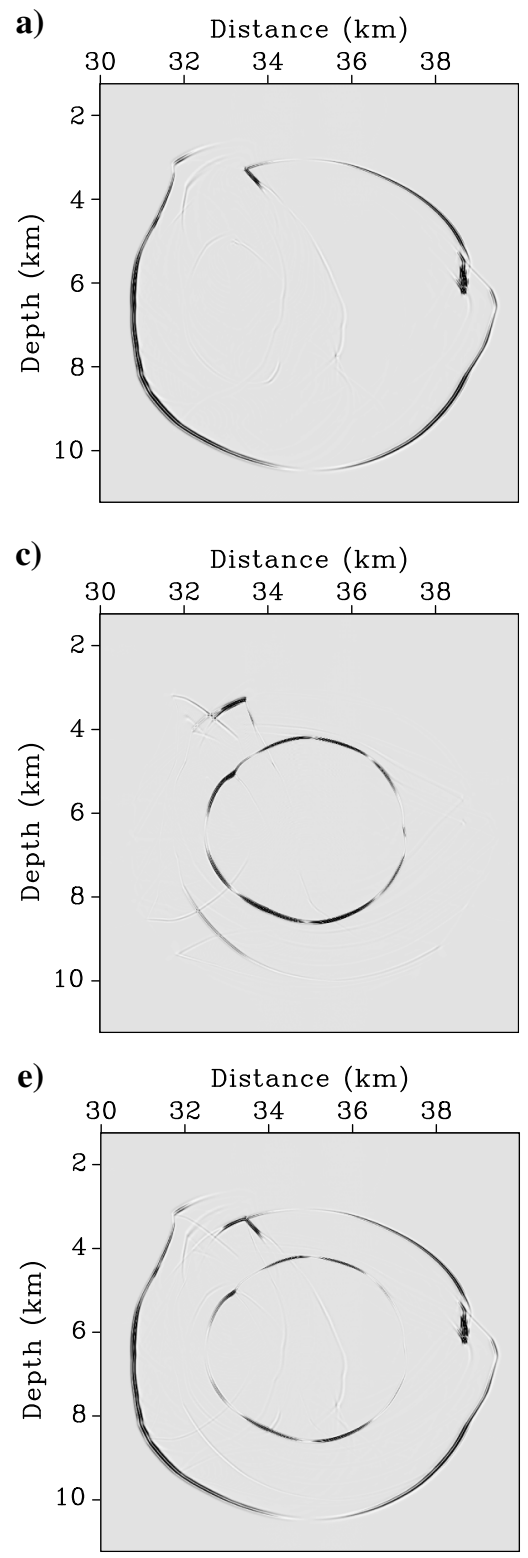

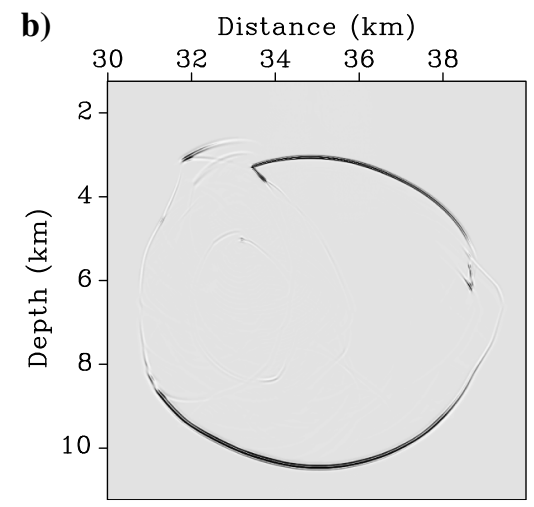

d)

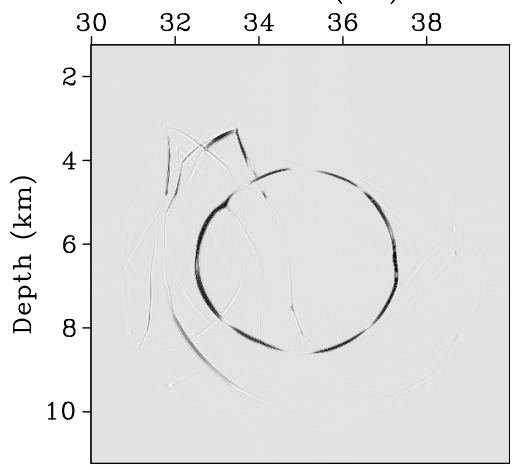

f)

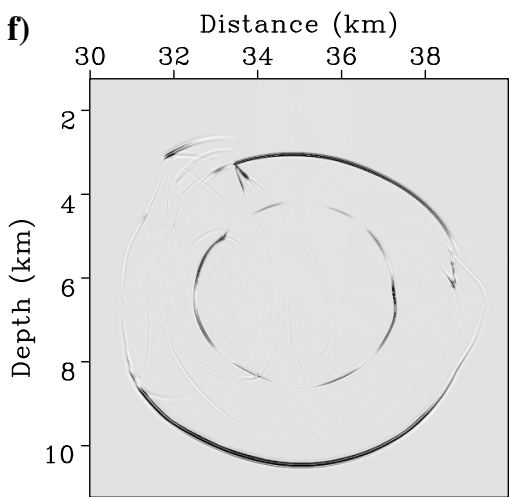




\section{CONCLUSIONS}

We have proposed a recursive integral method to simultaneously extrapolate and decompose the elastic wavefields on the base of second-order displacement equation for heterogeneous anisotropic media. The computational efficiency is guaranteed by merging the operations of time extrapolation and vector decomposition into a unified Fourier integral framework and speeding up the solutions using the low-rank approximation. The use of the $k$-space adjustment permits larger time steps without reducing accuracy or introducing instability in the low-rank pseudospectral scheme. The synthetic example shows that our method could produce dispersion-free decoupled and total elastic wavefields efficiently. We expect that the proposed approaches to extrapolate the decoupled elastic waves have great potential for applications such as elastic RTM and FWI of multicomponent seismic data acquired on land and at the ocean bottom. The focus for future work will be on the staggered-grid pseudospectral solution of the displacement- or velocity-stress equation for anisotropic media with strong heterogeneity and a lower order of symmetry.

\section{ACKNOWLEDGMENTS}

We would like to thank S. Fomel for sharing his experience in designing low-rank approximate algorithms for wave propagation. The first author appreciates T. F. Wang and J. Z. Sun for their useful discussion in this study. We acknowledge supports from the National Natural Science Foundation of China (no. 41474099) and Shanghai Natural Science Foundation (no. 14ZR1442900). This paper is also based upon work supported by the King Abdullah University of Science and Technology (KAUST) Office of Sponsored Research (OSR) under award no. 2230. We thank SEG, BP, and HESS Corporation for making the 2D VTI and TTI models available.

\section{APPENDIX A \\ COMPONENTS OF THE CHRISTOFFEL MATRIX}

For a general anisotropic medium, the components of the density normalized Christoffel matrix $\boldsymbol{\Gamma}$ are given as follows:

$$
\begin{aligned}
\Gamma_{11}= & {\left[C_{11} k_{x}^{2}+C_{66} k_{y}^{2}+C_{55} k_{z}^{2}+2 C_{56} k_{y} k_{z}\right.} \\
& \left.+2 C_{15} k_{x} k_{z}+2 C_{16} k_{x} k_{y}\right] / \rho, \\
\Gamma_{22}= & {\left[C_{66} k_{x}^{2}+C_{22} k_{y}^{2}+C_{44} k_{z}^{2}+2 C_{24} k_{y} k_{z}\right.} \\
& \left.+2 C_{46} k_{x} k_{z}+2 C_{26} k_{x} k_{y}\right] / \rho, \\
\Gamma_{33}= & {\left[C_{55} k_{x}^{2}+C_{44} k_{y}^{2}+C_{33} k_{z}^{2}+2 C_{34} k_{y} k_{z}\right.} \\
& \left.+2 C_{35} k_{x} k_{z}+2 C_{45} k_{x} k_{y}\right] / \rho, \\
\Gamma_{12}= & {\left[C_{16} k_{x}^{2}+C_{26} k_{y}^{2}+C_{45} k_{z}^{2}+\left(C_{46}+C_{25}\right) k_{y} k_{z}\right.} \\
& \left.+\left(C_{14}+C_{56}\right) k_{x} k_{z}+\left(C_{12}+C_{66}\right) k_{x} k_{y}\right] / \rho, \\
\Gamma_{13}= & {\left[C_{15} k_{x}^{2}+C_{46} k_{y}^{2}+C_{35} k_{z}^{2}+\left(C_{45}+C_{36}\right) k_{y} k_{z}\right.} \\
& \left.+\left(C_{13}+C_{55}\right) k_{x} k_{z}+\left(C_{14}+C_{56}\right) k_{x} k_{y}\right] / \rho, \Gamma_{23} \\
= & {\left[C_{56} k_{x}^{2}+C_{24} k_{y}^{2}+C_{34} k_{z}^{2}+\left(C_{44}+C_{23}\right) k_{y} k_{z}\right.} \\
& \left.+\left(C_{36}+C_{45}\right) k_{x} k_{z}+\left(C_{25}+C_{46}\right) k_{x} k_{y}\right] / \rho .
\end{aligned}
$$

\section{APPENDIX B}

\section{EXTENDED FORMULATIONS OF THE PSEUDO- SPECTRAL OPERATORS}

According to equations 6 and 8, we express the pseudospectral operator that can be used to extrapolate the coupled elastic wavefields in its extended formation as follows:

$$
\begin{aligned}
u_{x}(\mathbf{x}, t+\Delta t) & =-u_{x}(\mathbf{x}, t-\Delta t)+\int e^{i \mathbf{k} x} \mathbf{W}_{x x}(\mathbf{x}, \mathbf{k}) \tilde{u}_{x}(\mathbf{k}, t) d \mathbf{k} \\
& +\int e^{i \mathbf{k} \mathbf{x}} \mathbf{W}_{x y}(\mathbf{x}, \mathbf{k}) \tilde{u}_{y}(\mathbf{k}, t) d \mathbf{k} \\
& +\int e^{i \mathbf{k} \mathbf{x}} \mathbf{W}_{x z}(\mathbf{x}, \mathbf{k}) \tilde{u}_{z}(\mathbf{k}, t) d \mathbf{k}, \\
u_{y}(\mathbf{x}, t+\Delta t)= & -u_{y}(\mathbf{x}, t-\Delta t)+\int e^{i \mathbf{k} \mathbf{x}} \mathbf{W}_{x y}(\mathbf{x}, \mathbf{k}) \tilde{u}_{x}(\mathbf{k}, t) d \mathbf{k} \\
& +\int e^{i \mathbf{k} \mathbf{x}} \mathbf{W}_{y y}(\mathbf{x}, \mathbf{k}) \tilde{u}_{y}(\mathbf{k}, t) d \mathbf{k} \\
& +\int e^{i \mathbf{k x}} \mathbf{W}_{y z}(\mathbf{x}, \mathbf{k}) \tilde{u}_{z}(\mathbf{k}, t) d \mathbf{k}, \\
& =-u_{z}(\mathbf{x}, t-\Delta t)+\int e^{i \mathbf{k} \mathbf{x}} \mathbf{W}_{x z}(\mathbf{x}, \mathbf{k}) \tilde{u}_{x}(\mathbf{k}, t) d \mathbf{k} \\
& +\int e^{i \mathbf{k} \mathbf{x}} \mathbf{W}_{y z}(\mathbf{x}, \mathbf{k}) \tilde{u}_{y}(\mathbf{k}, t) d \mathbf{k} \\
& +\int e^{i \mathbf{k x}} \mathbf{W}_{z z}(\mathbf{x}, \mathbf{k}) \tilde{u}_{z}(\mathbf{k}, t) d \mathbf{k},
\end{aligned}
$$

where $\tilde{u}_{x}(\mathbf{k}, t), \tilde{u}_{y}(\mathbf{k}, t)$, and $\tilde{u}_{z}(\mathbf{k}, t)$ represent the three components of the elastic wavefields in wavenumber domain at the time of $t$.

For VTI or orthorhombic medium, we express the stiffness tensor as a Voigt matrix:

$$
\mathbf{C}=\left(\begin{array}{cccccc}
C_{11} & C_{12} & C_{13} & 0 & 0 & 0 \\
C_{12} & C_{22} & C_{23} & 0 & 0 & 0 \\
C_{13} & C_{23} & C_{33} & 0 & 0 & 00 \\
0 & 0 & 0 & C_{44} & 0 & 0 \\
0 & 0 & 0 & 0 & C_{55} & 0 \\
0 & 0 & 0 & 0 & 0 & C_{66}
\end{array}\right)
$$

where there are only five independent coefficient with $C_{12}=$ $C_{11}-2 C_{66}, C_{22}=C_{11}, C_{23}=C_{13}$, and $C_{55}=C_{44}$ for a VTI medium. Therefore, the propagation matrix has the following extended formulation:

$$
\begin{aligned}
& \mathbf{W}_{x x}(\mathbf{k})=2-\Delta t^{2}\left[C_{11} k_{x}^{2}+C_{66} k_{y}^{2}+C_{55} k_{z}^{2}\right], \\
& \mathbf{W}_{y y}(\mathbf{k})=2-\Delta t^{2}\left[C_{66} k_{x}^{2}+C_{22} k_{y}^{2}+C_{44} k_{z}^{2}\right], \\
& \mathbf{W}_{z z}(\mathbf{k})=2-\Delta t^{2}\left[C_{55} k_{x}^{2}+C_{44} k_{y}^{2}+C_{33} k_{z}^{2}\right], \\
& \mathbf{W}_{x y}(\mathbf{k})=-\Delta t^{2}\left[C_{12}+C_{66}\right] k_{x} k_{y}, \\
& \mathbf{W}_{x z}(\mathbf{k})=-\Delta t^{2}\left[C_{13}+C_{55}\right] k_{x} k_{z}, \\
& \mathbf{W}_{y z}(\mathbf{k})=-\Delta t^{2}\left[C_{23}+C_{44}\right] k_{y} k_{z} .
\end{aligned}
$$


If the principal axes of the medium are not aligned with the Cartesian axes, e.g., for the TTI and tilted orthorhombic media, we should apply the Bond transformation (Winterstein, 1990; Carcione, 2007) to get the stiffness matrix under the Cartesian system. This will introduce more mixed partial derivative terms in the wave equation, which demands a lot of computational effort if a FD algorithm is used to extrapolate the wavefields. Fortunately, for the pseudospectral solution, it only introduces negligible computation to prepare the propagation matrix and no extra computation for the wavefield extrapolation.

Similarly, we can write the propagation matrix $\overline{\mathbf{W}}_{i j}^{(m)}$ (in equation 20) for the decoupled elastic waves in its extended formulation as follows:

$$
\begin{aligned}
& \overline{\mathbf{W}}_{x x}^{(m)}(\mathbf{x}, \mathbf{k})=\mathbf{D}_{x x}^{(m)}(\mathbf{x}, \mathbf{k}) \mathbf{W}_{x x}(\mathbf{x}, \mathbf{k})+\mathbf{D}_{x y}^{(m)}(\mathbf{x}, \mathbf{k}) \mathbf{W}_{x y}(\mathbf{x}, \mathbf{k}) \\
& +\mathbf{D}_{x z}^{(m)}(\mathbf{x}, \mathbf{k}) \mathbf{W}_{x z}(\mathbf{x}, \mathbf{k}), \\
& \overline{\mathbf{W}}_{x y}^{(m)}(\mathbf{x}, \mathbf{k})=\mathbf{D}_{x x}^{(m)}(\mathbf{x}, \mathbf{k}) \mathbf{W}_{x y}(\mathbf{x}, \mathbf{k})+\mathbf{D}_{x y}^{(m)}(\mathbf{x}, \mathbf{k}) \mathbf{W}_{y y}(\mathbf{x}, \mathbf{k}) \\
& +\mathbf{D}_{x z}^{(m)}(\mathbf{x}, \mathbf{k}) \mathbf{W}_{y z}(\mathbf{x}, \mathbf{k}), \\
& \overline{\mathbf{W}}_{x z}^{(m)}(\mathbf{x}, \mathbf{k})=\mathbf{D}_{x x}^{(m)}(\mathbf{x}, \mathbf{k}) \mathbf{W}_{x z}(\mathbf{x}, \mathbf{k})+\mathbf{D}_{x y}^{(m)}(\mathbf{x}, \mathbf{k}) \mathbf{W}_{y z}(\mathbf{x}, \mathbf{k}) \\
& +\mathbf{D}_{x z}^{(m)}(\mathbf{x}, \mathbf{k}) \mathbf{W}_{z z}(\mathbf{x}, \mathbf{k}), \\
& \overline{\mathbf{W}}_{y x}^{(m)}(\mathbf{x}, \mathbf{k})=\mathbf{D}_{x y}^{(m)}(\mathbf{x}, \mathbf{k}) \mathbf{W}_{x x}(\mathbf{x}, \mathbf{k})+\mathbf{D}_{y y}^{(m)}(\mathbf{x}, \mathbf{k}) \mathbf{W}_{x y}(\mathbf{x}, \mathbf{k}) \\
& +\mathbf{D}_{y z}^{(m)}(\mathbf{x}, \mathbf{k}) \mathbf{W}_{x z}(\mathbf{x}, \mathbf{k}), \\
& \overline{\mathbf{W}}_{y y}^{(m)}(\mathbf{x}, \mathbf{k})=\mathbf{D}_{x y}^{(m)}(\mathbf{x}, \mathbf{k}) \mathbf{W}_{x y}(\mathbf{x}, \mathbf{k})+\mathbf{D}_{y y}^{(m)}(\mathbf{x}, \mathbf{k}) \mathbf{W}_{y y}(\mathbf{x}, \mathbf{k}) \\
& +\mathbf{D}_{y z}^{(m)}(\mathbf{x}, \mathbf{k}) \mathbf{W}_{y z}(\mathbf{x}, \mathbf{k}), \\
& \overline{\mathbf{W}}_{y z}^{(m)}(\mathbf{x}, \mathbf{k})=\mathbf{D}_{x y}^{(m)}(\mathbf{x}, \mathbf{k}) \mathbf{W}_{x z}(\mathbf{x}, \mathbf{k})+\mathbf{D}_{y y}^{(m)}(\mathbf{x}, \mathbf{k}) \mathbf{W}_{y z}(\mathbf{x}, \mathbf{k}) \\
& +\mathbf{D}_{y z}^{(m)}(\mathbf{x}, \mathbf{k}) \mathbf{W}_{z z}(\mathbf{x}, \mathbf{k}), \\
& \overline{\mathbf{W}}_{z x}^{(m)}(\mathbf{x}, \mathbf{k})=\mathbf{D}_{x z}^{(m)}(\mathbf{x}, \mathbf{k}) \mathbf{W}_{x x}(\mathbf{x}, \mathbf{k})+\mathbf{D}_{y z}^{(m)}(\mathbf{x}, \mathbf{k}) \mathbf{W}_{x y}(\mathbf{x}, \mathbf{k}) \\
& +\mathbf{D}_{z z}^{(m)}(\mathbf{x}, \mathbf{k}) \mathbf{W}_{x z}(\mathbf{x}, \mathbf{k}), \\
& \overline{\mathbf{W}}_{z y}^{(m)}(\mathbf{x}, \mathbf{k})=\mathbf{D}_{x z}^{(m)}(\mathbf{x}, \mathbf{k}) \mathbf{W}_{x y}(\mathbf{x}, \mathbf{k})+\mathbf{D}_{y z}^{(m)}(\mathbf{x}, \mathbf{k}) \mathbf{W}_{y y}(\mathbf{x}, \mathbf{k}) \\
& +\mathbf{D}_{z z}^{(m)}(\mathbf{x}, \mathbf{k}) \mathbf{W}_{y z}(\mathbf{x}, \mathbf{k}), \\
& \overline{\mathbf{W}}_{z z}^{(m)}(\mathbf{x}, \mathbf{k})=\mathbf{D}_{x z}^{(m)}(\mathbf{x}, \mathbf{k}) \mathbf{W}_{x z}(\mathbf{x}, \mathbf{k})+\mathbf{D}_{y z}^{(m)}(\mathbf{x}, \mathbf{k}) \mathbf{W}_{y z}(\mathbf{x}, \mathbf{k}) \\
& +\mathbf{D}_{z z}^{(m)}(\mathbf{x}, \mathbf{k}) \mathbf{W}_{z z}(\mathbf{x}, \mathbf{k}) \text {. }
\end{aligned}
$$

\section{APPENDIX C}

\section{THE $k$-SPACE ADJUSTMENT TO THE PSEUDOSPECTRAL SOLUTION}

According to the eigendecomposition of the Christoffel matrix (see equations 9-12), we can obtain the scalar wavefields for homogeneous anisotropic media using the theory of mode separation (Dellinger and Etgen, 1990) as follows:

$$
\hat{\bar{u}}_{i}=Q_{i j} \hat{u}_{j}
$$

where $\hat{\bar{u}}_{i}$ with $i=1,2,3$ represents the scalar $\mathrm{qP}, \mathrm{qS}_{1}$, and $\mathrm{qS}_{2}$ wavefields. Hence, these wavefields satisfy the same scalar wave equation

$$
\partial_{t t}^{2} \hat{\bar{u}}_{i}+\left(v_{i} k\right)^{2} \hat{\bar{u}}_{i}=0
$$

The standard leapfrog scheme for this equation can be expressed as follows:

$$
\frac{\hat{\bar{u}}_{i}^{(n+1)}-2 \hat{\bar{u}}_{i}^{(n)}+\hat{\bar{u}}_{i}^{(n-1)}}{\Delta t^{2}}=-\lambda_{i}^{2} \hat{\bar{u}}_{i}^{(n)} .
$$

It is well known that this solution is limited to small time steps for stable wave propagation.

Fortunately, there is an exact time-stepping solution for the second-order time derivatives allowing for any size of time steps for a homogeneous medium (Cox et al., 2007; Etgen and BrandsbergDahl, 2009); namely,

$$
\frac{\hat{\bar{u}}_{i}^{(n+1)}-2 \hat{\bar{u}}_{i}^{(n)}+\hat{\bar{u}}_{i}^{(n-1)}}{\Delta t^{2}}=\frac{-\sin ^{2}\left(\lambda_{i} \Delta t / 2\right)}{(\Delta t / 2)^{2}} \hat{\bar{u}}_{i}^{(n)} .
$$

Comparing equations $\mathrm{C}-3$ and $\mathrm{C}-4$ shows that it is possible to extend the length of a time step without reducing the accuracy by replacing $\left(\lambda_{i} \Delta t / 2\right)^{2}$ with $\sin ^{2}\left(\lambda_{i} \Delta t / 2\right)$. This opens up a possibility of replacing $k^{2}$ with $k^{2} \operatorname{sinc}^{2}\left(\lambda_{i} \Delta t / 2\right)$ as a $k$-space adjustment to the spatial derivatives, which may convert the time-stepping pseudospectral solution into an exact one for homogeneous media and stable for larger time steps (for a given level of accuracy) in heterogeneous media (Bojarski, 1982).

Nowadays, the $k$-space scheme is widely used to improve the approximation of the temporal derivative in acoustic and ultrasound (Tabei et al., 2002; Cox et al., 2007; Fang et al., 2014). As far as we know, Liu (1995) first applies $k$-space ideas to elastic wave problems. He derives a $k$-space form of the dyadic Green's function for the second-order wave equation and uses it to calculate the scattered field iteratively in a Born series. Firouzi et al. (2012) propose a $k$-space scheme on the base of the first-order elastic wave equation for isotropic media.

Accordingly, we apply the $k$-space adjustment to improve the performance of our two-step time-marching pseudospectral solution of the anisotropic elastic wave equation. To propagate the elastic waves on the base of equations 6 and 8 , we need to modify the eigenvalues of Christoffel matrix as in equation 14 .

\section{REFERENCES}

Aki, K., and P. Richards, 1980, Quantitative seismology, 2nd ed.: University Science Books.

Bale, R. A., 2003, Modeling 3D anisotropic elastic data using the pseudospectral approach: 65th Annual International Conference and Exhibition, EAGE, Extended Abstracts, C-43.

Bojarski, N. N., 1982, The k-space formulation of the scattering problem in the time domain: Journal of Acoustic Society of America, 72, 570-584, doi: $10.1121 / 1.388038$.

Carcione, J. M., 1999, Staggered mesh for the anisotropic and viscoelastic wave equation: Geophysics, 64, 1863-1866, doi: 10.1190/1.1444692.

Carcione, J. M., 2007, Wave fields in real media: Wave propagation in anisotropic, anelastic, porous and electromagnetic media: Elsevier Ltd.

Cheng, J. B., and S. Fomel, 2014, Fast algorithms of elastic wave mode separation and vector decomposition using low-rank approximation for anisotropic media: Geophysics, 79, no. 4, C97-C110, doi: 10.1190/ geo2014-0032.1.

Cheng, J. B., and W. Kang, 2014, Simulating propagation of separated wave modes in general anisotropic media, Part I: P-wave propagators: Geophysics, 79, no. 1, C1-C18, doi: 10.1190/geo2012-0504.1.

Chu, C., B. Macy, and P. Anno, 2011, An accurate and stable wave equation for pure acoustic TTI modeling: 81 st Annual International Meeting, SEG, Expanded Abstracts, 179-184. 
Cox, B. T., S. Kara, S. R. Arridge, and P. C. Beard, 2007, K-space propagation models for acoustically heterogeneous media: Application to biomedical photoacoustics: Journal of Acoustic Society of America, 121, 3453-3464, doi: 10.1121/1.2717409.

Crampin, S., 1991, Effects of point singularities on shear-wave propagation in sedimentary basin: Geophysical Journal International, 107, 531-543, doi: 10.1111/j.1365-246X.1991.tb01413.x.

Dablain, M. A., 1986, The application of high-order differencing to the scalar wave equation: Geophysics, 51, 54-66, doi: 10.1190/1.1442040.

Dellinger, J., and J. Etgen, 1990, Wavefield separation in two-dimensional anisotropic media: Geophysics, 55, 914-919, doi: 10.1190/1.1442906.

Du, X., P. J. Fowler, and R. P. Fletcher, 2014, Recursive integral timeextrapolation methods for waves: A comparative review: Geophysics, 79, no. 1, T9-T26, doi: 10.1190/geo2013-0115.1.

Engquist, B., and L. Ying, 2009, A fast directional algorithm for high frequency acoustic scattering in two dimensions: Communications Mathematical Sciences, 7, 327-345, doi: 10.4310/CMS.2009.v7.n2.a3.

Etgen, J., and S. Brandsberg-Dahl, 2009, The pseudo-analytical method: Application of pseudo-Laplacians to acoustic and acoustic anisotropic wave propagation: 79th Annual International Meeting, SEG, Expanded Abstracts, 2552-2556.

Fang, G., S. Fomel, Q. Z. Du, and J. W. Hu, 2014, Lowrank seismic wave extrapolation on a staggered grid: Geophysics, 79, no. 3, T157-T168, doi: 10.1190/geo2013-0290.1.

Firouzi, K., B. T. Cox, B. E. TYeeby, and N. Saffari, 2012, A first-order kspace model for elastic wave propagation in heterogeneous media: Journal of Acoustic Society of America, 132, 1271-1283, doi: 10.1121/1.4730897.

Fomel, S., L. Ying, and X. Song, 2013, Seismic wave extrapolation using lowrank symbol approximation: Geophysical Prospecting, 61, 526-536, doi: $10.1111 / \mathrm{j} .1365-2478.2012 .01064 . x$.

Fowler, P., and R. King, 2011, Modeling and reverse time migration of orthorhombic pseudoacoustic P-waves: 81st Annual International Meeting, SEG, Expanded Abstracts, 190-195.

Kang, W., and J. B. Cheng, 2012, Propagating pure wave modes in 3D general anisotropic media, Part II: SV and SH wave: 82nd Annual International Meeting, SEG, Expanded Abstracts, 1234-1238.

Kosloff, D., A. Q. Filho, E. Tessmer, and A. Behle, 1989, Numerical solution of the acoustic and elastic wave equation a new rapid expansion method: Geophysical Prospecting, 37, 383-394, doi: 10.1111/j.1365-2478.1989. tb02212.x.

Liu, F., S. Morton, S. Jiang, L. Ni, and J. Leveille, 2009, Decoupled wave equations for P and SV waves in an acoustic VTI media: 79th Annual International Meeting, SEG, Expanded Abstracts, 2844-2848.

Liu, Q., 1995, Generalization of the k-space formulation to elastodynamic scattering problems: Journal of Acoustic Society of America, 97, 13731379, doi: $10.1121 / 1.412079$

Liu, Y., and C. Li, 2000, Study of elastic wave propagation in two-phase anisotropic media by numerical modeling of pseudospectral method: Acta Seismologica Sinica, 13, 143-150, doi: 10.1007/s11589-000-0003-1.

Ma, D. T., and G. M. Zhu, 2003, P- and s-wave separated elastic wave equation numerical modeling (in Chinese): Oil Geophysical Prospecting, 38, 482-486.
Ozdenvar, T., and G. McMechan, 1996, Causes and reduction of numerical artifacts in pseudo-spectral wavefield extrapolation: Geophysical Journal International, 126, 819-828, doi: 10.1111/j.1365-246X.1996.tb04705.x.

Schoenberg, M., and K. Helbig, 1997, Orthorhombic media: Modeling elastic wave behavior in a vertically fractured earth: Geophysics, 62, 19541974, doi: 10.1190/1.1444297.

Song, X., and T. Alkhalifah, 2013, Modeling of pseudoacoustic p-waves in orthorhombic media with a low-rank approximation: Geophysics, 78, no. 4, C33-C40, doi: 10.1190/geo2012-0144.1.

Sun, J., and S. Fomel, 2013, Low-rank one-step wave extrapolation: 83rd Annual International Meeting, SEG, Expanded Abstracts, 1123-1127.

Sun, R., and G. A. McMechan, 2001, Scalar reverse-time depth migration of prestack elastic seismic data: Geophysics, 66, 1514-1527.

Tabei, M., T. D. Mast, and R. C. Waag, 2002, A k-space method for coupled first-order acoustic propagation equations: Journal of Acoustic Society of America, 111, 56-63.

Tsvankin, I., 2001, Seismic signatures and analysis of reflection data in anisotropic media: Elsevier Science Ltd.

Wang, T. F., and J. B. Cheng, 2015, Elastic wave mode decoupling for full waveform inversion: 85th Annual International Meeting, SEG, Expanded Abstracts, 1461-1466.

Wapenaar, C. P. A., N. A. Kinneging, and A. J. Berkhout, 1987, Principle of prestack migration based on the full elastic two-way wave equation: Geophysics, 52, 151-173, doi: 10.1190/1.1442291.

Winterstein, D., 1990, Velocity anisotropy terminology for geophysicists: Geophysics, 55, 1070-1088, doi: 10.1190/1.1442919.

Wu, Z., and T. Alkhalifah, 2014, The optimized expansion based low rank method for wavefield extrapolation: Geophysics, 79, no. 2, T51-T60, doi: 10.1190/geo2013-0174.1.

$\mathrm{Xu}, \mathrm{S}$, and H. Zhou, 2014, Accurate simulations of pure quasi-p-waves in complex anisotropic media: Geophysics, 79, no. 6, T341-T348, doi: 10 $.1190 / \mathrm{geo} 2014-0242.1$

Yan, J., and P. Sava, 2008, Isotropic angle domain elastic reverse time migration: Geophysics, 73, no. 6, S229-S239, doi: 10.1190/1.2981241.

Yan, J., and P. Sava, 2009, Elastic wave-mode separation for VTI media: Geophysics, 74, no. 5, WB19-WB32.

Yan, J., and P. Sava, 2011, Improving the efficiency of elastic wave-mode separation for heterogeneous tilted transverse isotropic media: Geophysics, 76, no. 4, T65-T78, doi: 10.1190/1.3581360.

Zhan, G., R. C. Pestana, and P. L. Stoffa, 2012, Decoupled equations for reverse time migration in tilted transversely isotropic media: Geophysics, 77, no. 2, T37-T45, doi: 10.1190/geo2011-0175.1.

Zhang, J., Z. Tian, and C. Wang, 2007, P- and s-wave separated elastic wave equation numerical modeling using $2 \mathrm{~d}$ staggered-grid: 77 th Annual International Meeting, SEG, Expanded Abstracts, 2104-2108.

Zhang, Q., and G. A. McMechan, 2010, 2D and 3D elastic wavefield vector decomposition in the wavenumber domain for VTI media: Geophysics, 75, no. 3, D13-D26, doi: 10.1190/1.3431045.

Zhang, Y., and G. Zhang, 2009, One-step extrapolation method for reverse time migration: Geophysics, 74, no. 4, A29-A33, doi: 10.1190/1 .3123476 . 